\title{
Asymptotics of solutions for a basic case of fluid structure interaction
}

\author{
Christoph Boeckle*† \\ Theoretical Physics Department \\ University of Geneva, Switzerland \\ christoph.boeckle@unige.ch
}

\author{
Peter Wittwer ${ }^{\ddagger}$ \\ Theoretical Physics Department \\ University of Geneva, Switzerland \\ peter.wittwer@unige.ch
}

May 9, 2022

\begin{abstract}
We consider the Navier-Stokes equations in a half-plane with a drift term parallel to the boundary and a small source term of compact support. We provide detailed information on the behavior of the velocity and the vorticity at infinity in terms of an asymptotic expansion at large distances from the boundary. The expansion is universal in the sense that it only depends on the source term through some multiplicative constants. This expansion is identical to the one for the problem of an exterior flow around a small body moving at constant velocity parallel to the boundary, and can be used as an artificial boundary condition on the edges of truncated domains for numerical simulations.
\end{abstract}

Keywords: Navier-Stokes equations; asymptotic expansions; exterior domain; fluid-structure interaction

\section{Contents}

1 Introduction 2

2 Functional framework 3

3 Functional equations 5

4 Asymptotic terms 7

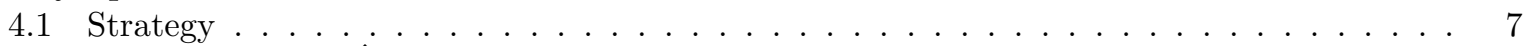

4.2 Leading order in $\hat{\psi}$ and $\hat{\varphi} \ldots \ldots \ldots \ldots \ldots \ldots \ldots \ldots$

4.3 Leading order in $\hat{\eta}$ and $\hat{\omega} \ldots \ldots \ldots \ldots \ldots \ldots \ldots$

4.4 Leading order in $\partial_{k} \hat{\omega} \ldots \ldots \ldots \ldots \ldots \ldots \ldots \ldots \ldots \ldots$

4.5 Improvement of the bounds on the non-linear terms . . . . . . . . . . . . . . . 17

4.5.1 New bounds. . . . . . . . . . . . . . . . . . . . 18

4.6 Second order in $\hat{\psi}$ and $\hat{\varphi} \ldots \ldots \ldots \ldots \ldots \ldots \ldots \ldots \ldots$

4.7 Final improvement of the bounds on $\hat{Q}_{0}, \hat{Q}_{1}$, and $\partial_{k} \hat{Q}_{1} \ldots \ldots \ldots \ldots$

4.8 Second order in $\hat{\eta}$ and $\hat{\omega} \ldots \ldots \ldots \ldots \ldots \ldots \ldots$

\begin{tabular}{ll} 
A Appendix & $\mathbf{2 7}$ \\
\hline
\end{tabular}

A.1 Explicit expressions for the asymptotes . . . . . . . . . . . . . . . . . . . . . . . . . .

A.2 Technical aspects of computations . . . . . . . . . . . . . . . . . 27

A.3 Bounds on convolution . . . . . . . . . . . . . . . . . . . . . . . 29

A.4 Convolution with the semi-groups $e^{\Lambda_{-} t}$ and $e^{-|k| t} \ldots \ldots \ldots \ldots \ldots$

\footnotetext{
*Supported by the Swiss National Science Foundation (Grant No. 200021-124403).

${ }^{\dagger}$ Corresponding author.

¥ Supported by the Swiss National Science Foundation (Grant No. 200021-124403).
} 


\section{Introduction}

In what follows, we study the steady Navier-Stokes equations in the half-plane $\Omega_{+}=\left\{(x, y) \in \mathbb{R}^{2} \mid y>1\right\}$ with a drift term parallel to the boundary, a force of compact support, and zero Dirichlet boundary conditions at the boundary of the half-plane and at infinity.

$$
\begin{aligned}
\partial_{x} \boldsymbol{u}+\boldsymbol{u} \cdot \nabla \boldsymbol{u}+\nabla p-\Delta \boldsymbol{u} & =\boldsymbol{F}, \\
\nabla \cdot \boldsymbol{u} & =0,
\end{aligned}
$$

where $\boldsymbol{F}$ is smooth and of compact support in $\Omega_{+}$, i.e., $\boldsymbol{F} \in C_{c}^{\infty}\left(\Omega_{+}\right)$, subject to the boundary conditions

$$
\begin{aligned}
\boldsymbol{u}(x, 1) & =0, \quad x \in \mathbb{R}, \\
\lim _{\mathbf{x} \rightarrow \infty} \boldsymbol{u}(\mathbf{x}) & =0 .
\end{aligned}
$$

For small forces, existence of a solution for this system together with basic bounds on the decay at infinity was proved in [9, and uniqueness of solutions was proved in [10 in a very general context. In [1] additional information on the decay at infinity was obtained. See [5], where the velocity field has been analyzed to leading order in a similar three dimensional case. For a general introduction to the method used in this series of papers, see [7].

Note that the asymptotic behavior is identical to the one for the problem of an exterior flow without force around a small body moving parallel to the wall at constant velocity described in a frame comoving with the body (see [10]). The explicit asymptotes of the unique solution to (11)-(4) may thus in particular be used as an artificial boundary condition for numerical simulations of the aforementioned flow with a body, see [2]. Artificial boundary conditions obtained this way have already been applied with success in the numerical resolution of two and three-dimensional flows in the full space (see [3, [4, [6] and [1]).

In the remainder of this paper, when we invoke "the solution", we refer to the solution constructed in [9], 1] and [10.

Our main result is summarized in the following theorem.

Theorem 1 Let $\boldsymbol{u}=(u, v)$ and $p$ be the solution to equations (1)-(4) for $\boldsymbol{F}$ small and let $\omega$ be the vorticity. Then, there exist constants $c_{1}, c_{2}$ such that for $\varepsilon>0$,

$$
\begin{aligned}
& \lim _{y \rightarrow \infty} \sup _{x \in \mathbb{R}}\left|y^{5 / 2-\varepsilon}\left(u(x, y)-u_{\text {as }}(x, y)\right)\right|=0, \\
& \lim _{y \rightarrow \infty} \sup _{x \in \mathbb{R}}\left|y^{5 / 2-\varepsilon}\left(v(x, y)-v_{\text {as }}(x, y)\right)\right|=0, \\
& \lim _{y \rightarrow \infty} \sup _{x \in \mathbb{R}}\left|y^{9 / 2-\varepsilon}\left(\omega(x, y)-\omega_{\text {as }}(x, y)\right)\right|=0,
\end{aligned}
$$

with

$$
\begin{aligned}
& u_{\text {as }}(x, y)=\frac{c_{1}}{y^{3 / 2}} \varphi_{1}(x / y)+\frac{c_{1}}{y^{2}} \varphi_{2,1}(x / y)+\frac{c_{2}}{y^{2}} \varphi_{2,2}(x / y)-\frac{c_{1}}{y^{2}} \eta_{W}\left(x / y^{2}\right)-\frac{c_{1}}{y^{3}} \eta_{B}\left(x / y^{2}\right), \\
& v_{\text {as }}(x, y)=\frac{c_{1}}{y^{3 / 2}} \psi_{1}(x / y)+\frac{c_{1}}{y^{2}} \psi_{2}(x / y)+\frac{c_{2}}{y^{2}} \psi_{2,2}(x / y)+\frac{c_{1}}{y^{3}} \omega_{W}\left(x / y^{2}\right)+\frac{c_{1}}{y^{4}} \omega_{B}\left(x / y^{2}\right), \\
& \omega_{\text {as }}(x, y)=\frac{c_{1}}{y^{3}} \omega_{W}\left(x / y^{2}\right)+\frac{c_{1}}{y^{4}} \omega_{B}\left(x / y^{2}\right),
\end{aligned}
$$

and functions $\varphi_{1}, \varphi_{2,1}, \varphi_{2,2}, \psi_{1}, \psi_{2,1}, \psi_{2,2}, \eta_{W}, \eta_{B}, \omega_{W}$ and $\omega_{B}$ as given in Appendix A.1.

Remark 2 This theorem is an immediate consequence of Theorem 10 in Section 3.

- The functions $\varphi_{1}, \varphi_{2,1}, \varphi_{2,2}, \psi_{1}, \psi_{2,1}, \psi_{2,2}, \eta_{W}, \eta_{B}, \omega_{W}$ and $\omega_{B}$ are universal, i.e., independent of $\boldsymbol{F}$.

- The power $5 / 2$ in the limits (5) and (6) is sharp, whereas the power $9 / 2$ in (7) can probably be improved by $1 / 2$ at the price of additional computations.

- Some terms in (8) and (9) are unimportant in view of the limits (5) and (6), but they are included such as to form a divergence-free velocity field in pairs of successive terms of $u_{\text {as }}$ and $v_{\text {as }}$ and such as to have two orders in both of the two scalings $x / y$ and $x / y^{2}$. 
- The explicit forms of $u_{\mathrm{as}}$ and $v_{\mathrm{as}}$ imply that

$$
\begin{aligned}
& \lim _{y \rightarrow \infty} y^{3 / 2} u(x y, y)=c_{1} \varphi_{1}(x), \\
& \lim _{y \rightarrow \infty} y^{3 / 2} v(x y, y)=c_{1} \psi_{1}(x),
\end{aligned}
$$

which shows that the bounds given in [9] are sharp. Moreover, the components of the velocity field associated to the functions $\varphi_{i}$ and $\psi_{i}$ are harmonic. The asymptotic expansion is thus given by the superposition of a potential flow and a flow carrying the vorticity, which is concentrated, to leading order, in a parabolic region called the "wake", in the sense that

$$
\lim _{y \rightarrow \infty} y^{3} \omega_{\mathrm{as}}\left(x y^{2}, y\right)=c_{1} \omega_{W}(x) .
$$

In contrast to the case of an exterior problem in $\mathbb{R}^{2}$ (see for example [4]), the vorticity is however not exponentially small outside the wake, since we have in particular, for all $x \in \mathbb{R}$,

$$
\lim _{y \rightarrow \infty} y^{4} \omega_{\mathrm{as}}(x, y)=c_{1} \omega_{B}(0) \neq 0,
$$

which shows that a background of vorticity is created by the interaction of the fluid with the boundary.

- This asymptotic expansion exhibits two scalings, whereas the three dimensional analogue (see [5]) exhibits only one (the analogue to the $x / y$ scaling). In addition, the current expansion is sharp for all components of the velocity field and takes into account an additional order, necessary to reveal the background of vorticity outside the wake.

- The constants $c_{1}$ and $c_{2}$ are expressed in terms of the solution, in (29) and (75) respectively.

- These results confirm the conjecture concerning the vorticity of the problem described in [8]. In the present paper the asymptotic behavior is known modulo the constants $c_{1}$ and $c_{2}$, whereas the conjecture had three undetermined constants in its representation.

The rest of this paper is organized as follows. In Section 2 we recall the functional framework defined in 9 in which the solutions were constructed. In Section 3 we also recall the map defined in 9 which yielded the solution in terms of its fixed point. We then present a new result which allows to improve the bounds on the solution. In Section 4 we first extract the leading order terms of the velocity and vorticity. Using these terms, we then improve the bounds from Section 3 and extract the next order of the asymptotic expansion. The appendix contains an explicit representation of the asymptotic terms, as well as various technical propositions and details of computations used in the main sections.

\section{Functional framework}

We first recall the functional framework of 9 .

Definition 3 Let $\hat{f}$ be a complex valued function on $\Omega_{+}$. Then, we define the inverse Fourier transform $f=\mathcal{F}^{-1}[\hat{f}]$ by the equation,

$$
f(x, y)=\mathcal{F}^{-1}[\hat{f}](x, y)=\frac{1}{2 \pi} \int_{\mathbb{R}} e^{-i k x} \hat{f}(k, y) d k,
$$

and $\hat{h}=\hat{f} * \hat{g}$ by

$$
\hat{h}(k, y)=(\hat{f} * \hat{g})(k, y)=\frac{1}{2 \pi} \int_{\mathbb{R}} \hat{f}\left(k-k^{\prime}, y\right) \hat{g}\left(k^{\prime}, y\right) d k^{\prime},
$$

whenever the integrals make sense. We note that for functions $f, g$ which are smooth and of compact support in $\Omega_{+}$we have $f=\mathcal{F}^{-1}[\hat{f}]$, and that $f g=\mathcal{F}^{-1}[\hat{f} * \hat{g}]$, where

$$
\hat{f}(k, y)=\mathcal{F}[f](k, y)=\int_{\mathbb{R}} e^{i k x} f(x, y) d x,
$$

and similarly $\hat{g}=\mathcal{F}[g]$. 
Whereas in direct space we use the variables $(x, y)$, in Fourier space we use the variables $(k, t)$, where $k$ is the Fourier-conjugated variable of $x$ and $y \equiv t$ (this choice of notation was made to remain consistent with [9]).

Definition 4 Let $\alpha, r \geq 0, k \in \mathbb{R}$ and $t \geq 1$, and let

$$
\mu_{\alpha, r}(k, t)=\frac{1}{1+\left(|k| t^{r}\right)^{\alpha}} .
$$

$W e$ set $\bar{\mu}_{\alpha}(k, t)=\mu_{\alpha, 1}(k, t), \tilde{\mu}_{\alpha}(k, t)=\mu_{\alpha, 2}(k, t)$.

Definition 5 We define, for fixed $\alpha \geq 0$, and $p, q \in \mathbb{R}, \mathcal{B}_{\alpha, p, q}$ to be the Banach space of functions $\hat{f} \in C(\mathbb{R} \backslash\{0\} \times[1, \infty), \mathbb{C})$, for which the norm

$$
\left\|\hat{f} ; \mathcal{B}_{\alpha, p, q}\right\|=\sup _{t \geq 1} \sup _{k \in \mathbb{R} \backslash\{0\}} \frac{|\hat{f}(k, t)|}{\frac{1}{t^{p}} \bar{\mu}_{\alpha}(k, t)+\frac{1}{t^{q}} \tilde{\mu}_{\alpha}(k, t)}
$$

is finite. The notations $\mathcal{B}_{\alpha, p, \infty}$ and $\mathcal{B}_{\alpha, \infty, q}$ are used for spaces of functions for which the norms

$$
\left\|\hat{f} ; \mathcal{B}_{\alpha, p, \infty}\right\|=\sup _{t \geq 1} \sup _{k \in \mathbb{R} \backslash\{0\}} \frac{|\hat{f}(k, t)|}{\frac{1}{t^{p}} \bar{\mu}_{\alpha}(k, t)}
$$

and

$$
\left\|\hat{f} ; \mathcal{B}_{\alpha, \infty, q}\right\|=\sup _{t \geq 1} \sup _{k \in \mathbb{R} \backslash\{0\}} \frac{|\hat{f}(k, t)|}{\frac{1}{t^{q}} \tilde{\mu}_{\alpha}(k, t)}
$$

are finite, respectively.

Remark 6 The following elementary properties of the spaces $\mathcal{B}_{\alpha, p, q}$ will be routinely used without mention:

- for $\alpha \geq 0$ and $p, q \in \mathbb{R}$, we have

$$
\mathcal{B}_{\alpha, p, q} \subset \mathcal{B}_{\alpha, \min \{p, q\}, \infty}
$$

- if $\alpha, \alpha^{\prime} \geq 0$, and $p, p^{\prime}, q, q^{\prime} \in \mathbb{R}$, then

$$
\mathcal{B}_{\alpha, p, q} \cap \mathcal{B}_{\alpha^{\prime}, p^{\prime}, q^{\prime}} \subset \mathcal{B}_{\min \left\{\alpha^{\prime}, \alpha,\right\}, \min \left\{p^{\prime}, p\right\}, \min \left\{q^{\prime}, q\right\}} .
$$

In the remainder of this paper, "const." stands for some constant independent of $k$ and $t$ that may change from one occurrence to the next without notice. If $\hat{f} \in \mathcal{B}_{\alpha, p, q}$ with $\alpha>1$, then we have the bound

$$
\begin{aligned}
\int_{\mathbb{R}}|\hat{f}(k, t)| d k & \leq\left\|\hat{f} ; \mathcal{B}_{\alpha, p, q}\right\| \int_{\mathbb{R}}\left(\frac{1}{t^{p}} \bar{\mu}_{\alpha}(k, t)+\frac{1}{t^{q}} \tilde{\mu}_{\alpha}(k, t)\right) d k \\
& \leq \text { const. }\left\|\hat{f} ; \mathcal{B}_{\alpha, p, q}\right\|\left(\frac{1}{t^{p+1}}+\frac{1}{t^{q+2}}\right) \\
& \leq \frac{\text { const. }}{t^{\min \{p+1, q+2)}\left\|\hat{f} ; \mathcal{B}_{\alpha, p, q}\right\|},
\end{aligned}
$$

which by Definition 3 immediately gives

$$
\sup _{x \in \mathbb{R}}|f(x, y)| \leq \frac{\text { const. }}{y^{\min \{p+1, q+2)}}\left\|\hat{f} ; \mathcal{B}_{\alpha, p, q}\right\|
$$

The $\mathcal{B}_{\alpha, p, q}$ spaces thus encode the decay behavior in direct space in the direction perpendicular to the wall, uniformly along lines parallel to the wall. For convenience later on we also define

$$
\begin{aligned}
\kappa & =\sqrt{k^{2}-i k}, \\
\tau & =t-1, \\
\sigma & =s-1,
\end{aligned}
$$


and

$$
\Lambda_{-}=-\operatorname{Re}(\kappa)=-\frac{1}{2} \sqrt{2 \sqrt{k^{2}+k^{4}}+2 k^{2}} .
$$

To further unburden the notations, we set

$$
\begin{aligned}
& \mu_{0}=\frac{1}{s^{7 / 2}} \bar{\mu}_{\alpha}(k, s)+\frac{1}{s^{3}} \tilde{\mu}_{\alpha}(k, s), \\
& \mu_{1}=\frac{1}{s^{7 / 2}} \bar{\mu}_{\alpha}(k, s)+\frac{1}{s^{4}} \tilde{\mu}_{\alpha}(k, s) .
\end{aligned}
$$

\section{Functional equations}

We recall the definition of the maps given in [9] which allowed to prove the existence of a solution by the contraction mapping principle. We begin by introducing the basic elements. The velocity field $(\hat{u}, \hat{v})$ is decomposed into

$$
\begin{aligned}
& \hat{u}=-\hat{\eta}+\hat{\varphi}, \\
& \hat{v}=\hat{\omega}+\hat{\psi},
\end{aligned}
$$

with $\hat{\omega}$ the vorticity. The nonlinear terms are represented by

$$
\begin{aligned}
& \hat{Q}_{0}(k, t)=\hat{u} * \hat{\omega}+\hat{F}_{2}, \\
& \hat{Q}_{1}(k, t)=\hat{v} * \hat{\omega}-\hat{F}_{1},
\end{aligned}
$$

where $\hat{\boldsymbol{F}}=\left(\hat{F}_{1}, \hat{F}_{2}\right)=\mathcal{F}[\boldsymbol{F}]$. The functions composing the velocity field are themselves further decomposed as follows

$$
\begin{aligned}
\hat{\psi} & =\sum_{m=0,1} \sum_{n=1,2,3} \hat{\psi}_{n, m}, & \hat{\varphi} & =\sum_{m=0,1} \sum_{n=1,2,3} \hat{\varphi}_{n, m}, \\
\hat{\omega} & =\sum_{m=0,1} \sum_{n=1,2,3} \hat{\omega}_{n, m}, & \hat{\eta} & =\sum_{m=0,1} \sum_{n=1,2,3} \hat{\eta}_{n, m} .
\end{aligned}
$$

For $\alpha>1$, we have the map

$$
\begin{array}{ccc}
\mathcal{N}: \mathcal{V}_{\alpha} & \rightarrow & \mathcal{V}_{\alpha}=\mathcal{B}_{\alpha, \frac{5}{2}, 1} \times \mathcal{B}_{\alpha, \frac{1}{2}, 0} \times \mathcal{B}_{\alpha, \frac{1}{2}, 1} \\
(\hat{\omega}, \hat{u}, \hat{v}) & \longmapsto & \mathcal{L}\left[\mathcal{C}[(\hat{\omega}, \hat{u}, \hat{v}),(\hat{\omega}, \hat{u}, \hat{v})]+\left(\hat{F}_{2},-\hat{F}_{1}\right)\right],
\end{array}
$$

with

$$
\begin{array}{ccc}
\mathcal{C}: & \mathcal{V}_{\alpha} \times \mathcal{V}_{\alpha} & \mathcal{W}_{\alpha}=\mathcal{B}_{\alpha, \frac{7}{2}, \frac{5}{2}} \times \mathcal{B}_{\alpha, \frac{7}{2}, \frac{5}{2}} \\
\left(\left(\hat{\omega}_{1}, \hat{u}_{1}, \hat{v}_{1}\right),\left(\hat{\omega}_{2}, \hat{u}_{2}, \hat{v}_{2}\right)\right) & \longmapsto & \left(\hat{u}_{1} * \hat{\omega}_{2}, \hat{v}_{1} * \hat{\omega}_{2}\right),
\end{array}
$$

a continuous bilinear map, and

$$
\begin{aligned}
& \mathcal{L}: \quad \mathcal{W}_{\alpha} \quad \rightarrow \quad \mathcal{V}_{\alpha} \\
& \left(\hat{Q}_{0}, \hat{Q}_{1}\right) \longmapsto \quad(\hat{\omega}, \hat{u}, \hat{v}),
\end{aligned}
$$

a continuous linear map. The solution $(\hat{\omega}, \hat{u}, \hat{v})$ is obtained, for $\left\|\left(\hat{F}_{2}, \hat{F}_{1}\right) ; \mathcal{W}_{\alpha}\right\|$ sufficiently small, as a fixed point of the map $\mathcal{N}$. Due to an improved bound given in Appendix A.3. tighter bounds on the nonlinear terms $\hat{Q}_{0}$ and $\hat{Q}_{1}$ can be obtained.

Proposition 7 Let $\alpha>1$. The bilinear map

$$
\begin{array}{ccc}
\mathcal{C}: & \mathcal{V}_{\alpha} \times \mathcal{V}_{\alpha} & \mathcal{Z}_{\alpha}=\mathcal{B}_{\alpha, \frac{7}{2}, 3} \times \mathcal{B}_{\alpha, \frac{7}{2}, 4} \\
\left(\left(\hat{\omega}_{1}, \hat{u}_{1}, \hat{v}_{1}\right),\left(\hat{\omega}_{2}, \hat{u}_{2}, \hat{v}_{2}\right)\right) & \longmapsto & \left(\hat{u}_{1} * \hat{\omega}_{2}, \hat{v}_{1} * \hat{\omega}_{2}\right),
\end{array}
$$

is continuous.

Proof. This is an immediate consequence of using Proposition 17 of the present paper instead of Proposition 9 in 9 in the proof of Lemma 4 in 9 .

Using Proposition 7 most of the bounds on the functions in (16) and (17) proved in [9] are easily improved. In the following proposition, we indicate in bold face all the indices which have changed with respect to Propositions 12, 14, 16 and 18 of 9 . 
Proposition 8 Let $\alpha>1, \delta>0$. We then have

$$
\begin{array}{llll}
\hat{\psi}_{1,0} \in \mathcal{B}_{\alpha, \frac{3}{2}-\boldsymbol{\delta}, \mathbf{2}} & \hat{\psi}_{1,1} \in \mathcal{B}_{\alpha, \frac{1}{2}, \mathbf{3}} & \hat{\varphi}_{1,0} \in \mathcal{B}_{\alpha, \frac{3}{2}-\boldsymbol{\delta}, \mathbf{2}} & \hat{\varphi}_{1,1} \in \mathcal{B}_{\alpha, \frac{1}{2}, \mathbf{3}} \\
\hat{\psi}_{2,0} \in \mathcal{B}_{\alpha, \frac{5}{2}, \mathbf{2}} & \hat{\psi}_{2,1} \in \mathcal{B}_{\alpha, \frac{5}{2}, \mathbf{3}} & \hat{\varphi}_{2,0} \in \mathcal{B}_{\alpha, \frac{5}{2}, \mathbf{2}} & \hat{\varphi}_{2,1} \in \mathcal{B}_{\alpha, \frac{5}{2}, \mathbf{3}} \\
\hat{\psi}_{3,0} \in \mathcal{B}_{\alpha, \frac{5}{2}, \mathbf{2}} & \hat{\psi}_{3,1} \in \mathcal{B}_{\alpha, \frac{5}{2}, \mathbf{3}} & \hat{\varphi}_{3,0} \in \mathcal{B}_{\alpha, \frac{5}{2}, \mathbf{2}} & \hat{\varphi}_{3,1} \in \mathcal{B}_{\alpha, \frac{5}{2}, \mathbf{3}} \\
\hat{\omega}_{1,0} \in \mathcal{B}_{\alpha, \frac{7}{2}, \mathbf{3}-\boldsymbol{\delta}} & \hat{\omega}_{1,1} \in \mathcal{B}_{\alpha, \frac{5}{2}, 1} & \hat{\eta}_{1,0} \in \mathcal{B}_{\alpha, \frac{5}{2}, \mathbf{2}-\boldsymbol{\delta}} & \hat{\eta}_{1,1} \in \mathcal{B}_{\alpha, \frac{3}{2}, 0} \\
\hat{\omega}_{2,0} \in \mathcal{B}_{\alpha, \infty, 3} & \hat{\omega}_{2,1} \in \mathcal{B}_{\alpha, \frac{5}{2}, \mathbf{3}} & \hat{\eta}_{2,0} \in \mathcal{B}_{\alpha, \infty, 2} & \hat{\eta}_{2,1} \in \mathcal{B}_{\alpha, \frac{5}{2}, \mathbf{3}} \\
\hat{\omega}_{3,0} \in \mathcal{B}_{\alpha, \frac{7}{2}, \mathbf{3}} & \hat{\omega}_{3,1} \in \mathcal{B}_{\alpha, \frac{5}{2}, \mathbf{3}} & \hat{\eta}_{3,0} \in \mathcal{B}_{\alpha, \frac{5}{2}, \mathbf{2}} & \hat{\eta}_{3,1} \in \mathcal{B}_{\alpha, \frac{3}{2}, \mathbf{2}}
\end{array}
$$

Remark 9 Given the decay behavior in direct space provided by (11), it is clear that the components with indices $(1,1)$ play a dominant role in Theorem 1, In fact, functions in $\mathcal{B}_{\alpha, p, q}$ with $p \geq 3 / 2$ and $q \geq 1 / 2$ are negligible in the sense of the limits given in (5) and (6), although Theorem 1 includes some additional terms to satisfy the divergence-free criterion and to have two orders of the asymptotics in both scalings. In the same way, functions with indices $p \geq 4$ and $q \geq 3$ are negligible in the sense of the limit given in (7). One would then expect $\hat{\omega}_{2,1}$ and $\hat{\omega}_{3,1}$ to be relevant, but new and better bounds are proved in Section 4.7, so that they will turn out to be negligible, too.

Proof. Using that $\left(\hat{Q}_{0}, \hat{Q}_{1}\right) \in \mathcal{Z}_{\alpha}$ and following otherwise the proof of Lemma 5 in [9], this is straightforward for all functions except $\hat{\omega}_{2,0}, \hat{\eta}_{2,0}$ and $\hat{\omega}_{3,0}$. Note that $\delta \in(0,1)$ using (118).

For $\hat{\omega}_{2,0}$, we recall that

$$
\hat{\omega}_{2,0}(k, t)=\frac{1}{2} e^{-\kappa(t-1)} \int_{t}^{\infty} f_{2,0}(k, s-1) \hat{Q}_{0}(k, s) d s,
$$

with

$$
f_{2,0}(k, \sigma)=\left(\frac{i k}{\kappa}-\frac{(|k|+\kappa)^{2}}{\kappa}\right) e^{-\kappa \sigma}+2(|k|+\kappa) e^{-|k| \sigma}
$$

We have the bound

$$
\left|f_{2,0}(k, \sigma)\right| \leq \text { const. }\left(|k|^{1 / 2}+|k|\right) e^{-|k| \sigma},
$$

so that we therefore have for $\hat{\omega}_{2,0}$

$$
\begin{aligned}
\left|\hat{\omega}_{2,0}(k, t)\right| & \leq \text { const. } e^{\Lambda_{-}(t-1)} \int_{t}^{\infty}\left|f_{2,0}(k, \sigma)\right| \mu_{0}(k, s) d s \\
& \leq \text { const. } e^{\Lambda_{-}(t-1)} e^{|k|(t-1)} \int_{t}^{\infty}\left(|k|^{1 / 2}+|k|\right) e^{-|k| \sigma} \frac{1}{s^{7 / 2}} \bar{\mu}_{\alpha}(k, s) d s \\
& + \text { const. } e^{\Lambda_{-}(t-1)} e^{|k|(t-1)} \int_{t}^{\infty}\left(|k|^{1 / 2}+|k|\right) e^{-|k| \sigma} \frac{1}{s^{3}} \tilde{\mu}_{\alpha}(k, s) d s .
\end{aligned}
$$

The term in (20) is estimated with Proposition 24

$$
e^{\Lambda_{-}(t-1)} e^{|k|(t-1)} \int_{t}^{\infty}\left(|k|^{1 / 2}+|k|\right) e^{-|k| \sigma} \frac{1}{s^{7 / 2}} \bar{\mu}_{\alpha}(k, s) d s \leq \text { const. } e^{\Lambda_{-}(t-1)} \frac{1}{t^{3}} \bar{\mu}_{\alpha}(k, t) .
$$

The term (21) requires us to distinguish the cases $1 \leq t \leq 2$ and $t>2$. In the first case, we have, using Proposition 24,

$$
e^{\Lambda_{-}(t-1)} e^{|k|(t-1)} \int_{t}^{\infty}\left(|k|^{1 / 2}+|k|\right) e^{-|k| \sigma} \frac{1}{s^{3}} \tilde{\mu}_{\alpha}(k, s) d s \leq \text { const. } \frac{1}{t^{5 / 2}} \tilde{\mu}_{\alpha}(k, t) \leq \text { const. } \frac{1}{t^{3}} \tilde{\mu}_{\alpha}(k, t),
$$

and in the second case we have, using (114) to trade the factor $|k|^{1 / 2}$ for a factor $s^{-1}$ and then applying Proposition 24,

$$
\begin{aligned}
& e^{\Lambda_{-}(t-1)} e^{|k|(t-1)} \int_{t}^{\infty}\left(|k|^{1 / 2}+|k|\right) e^{-|k| \sigma} \frac{1}{s^{3}} \tilde{\mu}_{\alpha}(k, s) d s \\
& \leq \text { const. } e^{\Lambda_{-}(t-1)} e^{|k|(t-1)} \int_{t}^{\infty} e^{-|k| \sigma}\left(\frac{1}{s^{4}} \tilde{\mu}_{\alpha-1 / 2}(k, s)+|k| \frac{1}{s^{3}} \tilde{\mu}_{\alpha}(k, s)\right) d s \\
& \leq \text { const. } e^{\Lambda_{-}(t-1)} \frac{1}{t^{3}} \tilde{\mu}_{\alpha-1 / 2}(k, t) .
\end{aligned}
$$


Collecting (22)-(24) and applying (113), we finally have

$$
\left|\hat{\omega}_{2,0}(k, t)\right| \leq \text { const. } \frac{1}{t^{3}} \tilde{\mu}_{\alpha}(k, t) .
$$

Indeed, for $t>2$ the index $\alpha$ is arbitrarily large due to the exponential factor.

For the function $\hat{\eta}_{2,0}$, we have, from [9] and using Proposition 7, that

$$
\left|\hat{\eta}_{2,0}(k, t)\right| \leq \text { const. } e^{\Lambda_{-}(t-1)} e^{-|k|(t-1)}\left(\frac{1}{t^{5 / 2}} \bar{\mu}_{\alpha}(k, t)+\frac{1}{t^{2}} \tilde{\mu}_{\alpha}(k, t)\right) .
$$

Using inequality (113) shows that $\hat{\eta}_{2,0} \in \mathcal{B}_{\alpha, \infty, 2}$.

For $\hat{\omega}_{3,0}$ we recall from [9] that

$$
\left|\hat{\omega}_{3,0}(k, t)\right| \leq \text { const. }\left|\frac{\kappa}{i k}\left(e^{\kappa(t-1)}-e^{-\kappa(t-1)}\right)\right| \int_{t}^{\infty}\left|f_{3,0}(k, \sigma)\right| \mu_{0}(k, s) d s,
$$

with

$$
\left|f_{3,0}(k, \sigma)\right| \leq \text { const. } e^{\Lambda_{-} \sigma} \min \left\{1,\left|\Lambda_{-}\right|^{2}\right\} \leq \text { const. } e^{\Lambda_{-} \sigma}\left|\Lambda_{-}\right| .
$$

Since $\left|\Lambda_{-}\right| \sim|k|^{1 / 2}$ for $|k| \leq 1$ and $\left|\Lambda_{-}\right| \sim|k|$ for $|k|>1$, we use for the first case $\left|f_{3,0}(k, \sigma)\right| \leq$ const. $e^{\Lambda_{-} \sigma}\left|\Lambda_{-}\right|^{2}$ and for the second case $\left|f_{3,0}(k, \sigma)\right| \leq$ const. $e^{\Lambda_{-} \sigma}\left|\Lambda_{-}\right|$and we have, for all $|k|$, using Proposition 21,

$$
\begin{aligned}
\left|\hat{\omega}_{3,0}(k, t)\right| & \leq \text { const. } e^{\left|\Lambda_{-}\right|(t-1)} \int_{t}^{\infty}\left|\Lambda_{-}\right| \mu_{0}(k, s) d s \\
& \leq \text { const. }\left(\frac{1}{t^{7 / 2}} \bar{\mu}_{\alpha}(k, t)+\frac{1}{t^{3}} \tilde{\mu}_{\alpha}(k, t)\right) .
\end{aligned}
$$

\section{Asymptotic terms}

\subsection{Strategy}

In this section we extract the leading asymptotic terms of the functions $\hat{\psi}, \hat{\varphi}, \hat{\eta}, \hat{\omega}$ and $\partial_{k} \hat{\omega}$. We then calculate an explicit representation of these asymptotic terms in direct space which allows us to prove even tighter bounds on the nonlinear terms $\hat{Q}_{0}, \hat{Q}_{1}$ as well as $\partial_{k} \hat{Q}_{1}$, than the ones given in Proposition[7 and [1]. The new bounds on $\hat{Q}_{0}$ and $\hat{Q}_{1}$ are then used to further improve the bounds on $\hat{\psi}, \hat{\varphi}, \hat{\eta}$, and $\hat{\omega}$, which, together with the tighter bound on $\partial_{k} \hat{Q}_{1}$, allow us to extract second-order terms in two steps. First, we extract the second order terms of $\hat{\psi}$ and $\hat{\varphi}$, which allows us to improve the bounds on the non-linear terms once again using their direct-space representation. Then, we proceed to extract the second order terms of $\hat{\eta}$ and $\hat{\omega}$.

The extraction procedure is as follows: we first identify the leading components in view of Proposition 8 and Remark 9 . We then calculate for each of these components the pointwise limit as $t \rightarrow \infty$ for one of two scalings: $k \mapsto k / t$ if the slowest direct space decay in the sense of (11) is due to the index $p$, $k \mapsto k / t^{2}$ if it is due to the index $q$. We finally prove that the difference between the leading component and this pointwise limit is in a $\mathcal{B}_{\alpha, p^{\prime}, q}$ or $\mathcal{B}_{\alpha, p, q^{\prime}}$ which is smaller due to an improvement in the index that determined the scaling choice, thus identifying the pointwise limit as the leading asymptotic term. For the second order asymptotic term, we proceed in the same way using any new bound obtained in between to identify the components from which we have to extract it. As we will see, this is actually the leading component minus the leading order asymptotic term, for which we then calculate a new pointwise limit to obtain the second order term

In this section, some bounds lead to a decrease of $\alpha$ by -3 . Since the solution exists for arbitrary $\alpha>3$, this does not pose a problem. We now present our main technical result. To unburden the notation in the proofs and results we set

$$
\begin{aligned}
\alpha^{\prime} & =\alpha-1, \\
\alpha^{\prime \prime} & =\alpha-2 .
\end{aligned}
$$


Theorem 10 (asymptotes in $\mathcal{B}_{\alpha, p, q}$ spaces) Let $\hat{u} \in \mathcal{B}_{\alpha, \frac{1}{2}, 0}, \hat{v} \in \mathcal{B}_{\alpha, \frac{1}{2}, 1}, \hat{\omega} \in \mathcal{B}_{\alpha, \frac{5}{2}, 1}$ as constructed in [9], with $\hat{u}=-\hat{\eta}+\hat{\varphi}, \hat{v}=\hat{\omega}+\hat{\psi}$. We then have, for $\alpha>4, \infty$ arbitrarily large and $\delta>0$,

$$
\begin{array}{ll}
\hat{\psi}-\hat{\psi}_{\mathrm{as}, 1} \in \mathcal{B}_{\alpha, 1, \infty} & \hat{\psi}-\hat{\psi}_{\mathrm{as}, 1}-\hat{\psi}_{\mathrm{as}, 2} \in \mathcal{B}_{\alpha, \frac{3}{2}-\delta, \infty} \\
\hat{\varphi}-\hat{\varphi}_{\mathrm{as}, 1} \in \mathcal{B}_{\alpha, 1, \infty} & \hat{\varphi}-\hat{\varphi}_{\mathrm{as}, 1}-\hat{\varphi}_{\mathrm{as}, 2} \in \mathcal{B}_{\alpha, \frac{3}{2}-\delta, \infty} \\
\hat{\omega}-\hat{\omega}_{\mathrm{as}, 1} \in \mathcal{B}_{\alpha, \frac{7}{2}-\delta, 2} & \hat{\omega}-\hat{\omega}_{\mathrm{as}, 1}-\hat{\omega}_{\mathrm{as}, 2} \in \mathcal{B}_{\alpha, \frac{7}{2}-\delta, 3-\delta} \\
\hat{\eta}-\hat{\eta}_{\mathrm{as}, 1} \in \mathcal{B}_{\alpha, \frac{5}{2}-\delta, 1} & \hat{\eta}-\hat{\eta}_{\mathrm{as}, 1}-\hat{\eta}_{\mathrm{as}, 2} \in \mathcal{B}_{\alpha, \frac{5}{2}-\delta, 2-\delta}
\end{array}
$$

where the functions with the subscripts "as" and "as, 2 " are given as follows: for $\hat{\psi}$ by (30) and (73), for $\hat{\varphi}$ by (31) and (74), for $\hat{\omega}$ by (42) and (90), and finally for $\hat{\eta}$ by (41) and (89).

In the remainder of this section we give a proof of this theorem.

\subsection{Leading order in $\hat{\psi}$ and $\hat{\varphi}$}

In view of Proposition 8 and Remark 9, the leading order term of $\hat{\psi}$ and $\hat{\varphi}$ are to be extracted from $\hat{\psi}_{1,1}$ and $\hat{\varphi}_{1,1}$, respectively. We use that $\hat{\psi}, \hat{\varphi} \in \mathcal{B}_{\alpha, \frac{1}{2}, \infty} \supset \mathcal{B}_{a, \frac{1}{2}, 2}$, since for these functions we are not interested in the wake behavior. We have (see [9]),

$$
\begin{aligned}
& \hat{\psi}_{1,1}(k, t)=\frac{1}{2} e^{-|k|(t-1)} \int_{1}^{t} h_{1,1}(k, s-1) \hat{Q}_{1}(k, s) d s, \\
& \hat{\varphi}_{1,1}(k, t)=\frac{1}{2} e^{-|k|(t-1)} \int_{1}^{t} k_{1,1}(k, s-1) \hat{Q}_{1}(k, s) d s,
\end{aligned}
$$

with

$$
\begin{aligned}
& h_{1,1}(k, \sigma)=-e^{|k| \sigma}+\frac{(|k|+\kappa)^{2}}{i k} e^{-|k| \sigma}-2 \frac{\kappa(|k|+\kappa)}{i k} e^{-\kappa \sigma}, \\
& k_{1,1}(k, \sigma)=-\frac{|k|}{i k} h_{1,1}(k, \sigma) .
\end{aligned}
$$

Formally, we get from (25) and (26)

$$
\begin{aligned}
& \lim _{t \rightarrow \infty} \sqrt{t} \hat{\psi}_{1,1}(k / t, t)=-c_{1} \sqrt{-i k} e^{-|k|}=: \hat{\psi}_{1,1}^{1}(k), \\
& \lim _{t \rightarrow \infty} \sqrt{t} \hat{\varphi}_{1,1}(k / t, t)=c_{1} \frac{|k|}{i k} \sqrt{-i k} e^{-|k|}=: \hat{\varphi}_{1,1}^{1}(k),
\end{aligned}
$$

with

$$
c_{1}=\int_{1}^{\infty}(s-1) \hat{Q}_{1}(0, s) d s .
$$

This motivates the definition of the functions

$$
\begin{aligned}
& \hat{\psi}_{\mathrm{as}, 1}(k, t)=\frac{1}{\sqrt{t}} \hat{\psi}_{1,1}^{1}(k t)=-c_{1} \sqrt{-i k} e^{-|k| t}, \\
& \hat{\varphi}_{\mathrm{as}, 1}(k, t)=\frac{1}{\sqrt{t}} \hat{\varphi}_{1,1}^{1}(k t)=c_{1} \frac{|k|}{i k} \sqrt{-i k} e^{-|k| t} .
\end{aligned}
$$

Note that $\hat{\psi}_{\text {as }, 1}, \hat{\varphi}_{\text {as }, 1} \in \mathcal{B}_{\alpha, \frac{1}{2}, \infty}$. We now show that

$$
\begin{aligned}
& \hat{\psi}_{1,1}-\hat{\psi}_{\mathrm{as}, 1} \in \mathcal{B}_{\alpha^{\prime}, 1, \infty} \\
& \hat{\varphi}_{1,1}-\hat{\varphi}_{\mathrm{as}, 1} \in \mathcal{B}_{\alpha^{\prime}, 1, \infty} .
\end{aligned}
$$

Proof. We have

$$
\hat{\psi}_{1,1}=-\frac{|k|}{i k} \hat{\varphi}_{1,1}
$$

and thus all the bounds on $\hat{\psi}_{1,1}$ are directly transposable to $\hat{\varphi}_{1,1}$, and we only present the proof for $\hat{\psi}_{1,1}$. In order to prove (32) we analyze

$$
\begin{aligned}
\hat{\psi}_{1,1}(k, t)-\hat{\psi}_{\mathrm{as}, 1}(k, t) & =\frac{1}{2} e^{-|k|(t-1)} \int_{1}^{t} h_{1,1}(k, s-1) \hat{Q}_{1}(k, s) d s \\
& +\frac{1}{2} e^{-|k| t} \int_{1}^{\infty} 2 \sqrt{-i k}(s-1) \hat{Q}_{1}(0, s) d s
\end{aligned}
$$


We rewrite this expression as a sum of terms which can easily be bounded. Namely,

$$
\hat{\psi}_{1,1}(k, t)-\hat{\psi}_{\mathrm{as}, 1}(\hat{\psi}, t)=\sum_{i=1}^{3} \hat{\psi}_{i}^{r, 1}
$$

with

$$
\begin{aligned}
\hat{\psi}_{1}^{r, 1} & =\frac{1}{2}\left(e^{-|k|(t-1)}-e^{-|k| t}\right) \int_{1}^{t} h_{1,1}(k, s-1) \hat{Q}_{1}(k, s) d s \\
\hat{\psi}_{2}^{r, 1} & =\frac{1}{2} e^{-|k| t} \int_{1}^{t}\left(h_{1,1}(k, s-1) \hat{Q}_{1}(k, s)+2 \sqrt{-i k}(s-1) \hat{Q}_{1}(0, s)\right) d s \\
\hat{\psi}_{3}^{r, 1} & =\frac{1}{2} e^{-|k| t} \int_{t}^{\infty} 2 \sqrt{-i k}(s-1) \hat{Q}_{1}(0, s) d s
\end{aligned}
$$

To bound $\hat{\psi}_{1}^{r, 1}$ we use that

$$
\left|h_{1,1}(k, \sigma)\right| \leq \text { const. }(1+|k|) e^{|k| \sigma} \min \left\{1,\left(1+|k|^{1 / 2}\right)|k|^{1 / 2} \sigma\right\},
$$

inequality (114), Propositions 22 and 23, so that we get

$$
\begin{aligned}
\left|\hat{\psi}_{1}^{r, 1}\right| & =\left|\frac{1}{2}\left(e^{-|k|(t-1)}-e^{-|k| t}\right) \int_{1}^{t} h_{1,1}(k, s-1) \hat{Q}_{1}(k, s) d s\right| \\
& \leq \text { const. } e^{-|k| t}|k| e^{|k|} \int_{1}^{t}(1+|k|) e^{|k| \sigma} \min \left\{1,\left(1+|k|^{1 / 2}\right)|k|^{1 / 2} \sigma\right\} \mu_{1}(k, s) d s \\
& \leq \text { const. }\left(\frac{1}{t^{3 / 2}} \bar{\mu}_{\alpha-1}(k, t)+\frac{1}{t^{4}} \tilde{\mu}_{\alpha-1}(k, t)\right)
\end{aligned}
$$

which shows that $\hat{\psi}_{1}^{r, 1} \in \mathcal{B}_{\alpha^{\prime}, \frac{3}{2}, \infty}$.

To bound $\hat{\psi}_{2}^{r, 1}$ we first note that by (108)

$$
\begin{aligned}
& h_{1,1}(k, \sigma) \hat{Q}_{1}(k, s)+2 \sqrt{-i k} \sigma \hat{Q}_{1}(0, s) \\
& =\left(h_{1,1}(k, \sigma)+2 \sqrt{-i k} \sigma\right) \hat{Q}_{1}(k, s)-2 \sqrt{-i k} k \sigma \partial_{k} \hat{Q}_{1}(\zeta, s),
\end{aligned}
$$

for some $\zeta \in[0, k]$. We analyze the expression

$$
h_{1,1}(k, \sigma)+2 \sqrt{-i k} \sigma=-e^{|k| \sigma}+\frac{(|k|+\kappa)^{2}}{i k} e^{-|k| \sigma}-2 \frac{\kappa(|k|+\kappa)}{i k} e^{-\kappa \sigma}+2 \sqrt{-i k} \sigma
$$

in further detail, with $h_{1,1}$ given by (27). A straightforward bound is

$$
\left|h_{1,1}(k, \sigma)+2 \sqrt{-i k} \sigma\right| \leq \text { const. }(1+|k|(\sigma+1)) e^{|k| \sigma},
$$

but since the leading terms cancel, we also have

$$
\begin{aligned}
h_{1,1}(k, \sigma)+2 \sqrt{-i k} \sigma & =-\left(e^{|k| \sigma}-1-|k| \sigma\right)-\left(e^{-|k| \sigma}-1+|k| \sigma\right)+2\left(e^{-\kappa \sigma}-1+\kappa \sigma\right) \\
& +\frac{2|k|^{2}+2|k| \kappa}{i k}\left(\left(e^{-|k| \sigma}-1\right)-\left(e^{-\kappa \sigma}-1\right)\right)-2 \kappa \sigma+2 \sqrt{-i k} \sigma
\end{aligned}
$$

which we can bound, using (110), by

$$
\begin{aligned}
\left|h_{1,1}(k, \sigma)+2 \sqrt{-i k} \sigma\right| & \leq \text { const. }|k|^{2} \sigma^{2} e^{|k| \sigma}+\text { const. }|k|^{2} \sigma^{2}+\text { const. }|\kappa|^{2} \sigma^{2} \\
& + \text { const. }\left(|k|^{1 / 2}+|k|\right)(|k| \sigma+|\kappa| \sigma)+\text { const. }|k|^{3 / 2} \sigma \\
& \leq \text { const. } \sigma(\sigma+1)\left(|k|+|k|^{2}\right) e^{|k| \sigma} .
\end{aligned}
$$

We have used here, and shall routinely use again throughout this paper without further explicit mention, that for all $z \in \mathbb{C}$ with $\operatorname{Re}(z) \leq 0$ and $N \in \mathbb{N}_{0}$,

$$
\left|\frac{e^{z}-\sum_{n=0}^{N} \frac{1}{n !} z^{n}}{z^{N+1}}\right| \leq \text { const. }
$$


and for all $z \in \mathbb{C}$ with $\operatorname{Re}(z)>0$

$$
\left|\frac{e^{z}-\sum_{n=0}^{N} \frac{1}{n !} z^{n}}{z^{N+1}}\right| \leq \text { const. } e^{\operatorname{Re}(z)} .
$$

Therefore, using (34) and (35), we get

$$
\left|h_{1,1}(k, \sigma)+2 \sqrt{-i k} \sigma\right| \leq \text { const. } \min \left\{(1+|k|(\sigma+1)),\left(|k|+|k|^{2}\right) \sigma(\sigma+1)\right\} e^{|k| \sigma} .
$$

Collecting these bounds yields

$$
\begin{aligned}
\left|\hat{\psi}_{2}^{r, 1}\right| & =\left|\frac{1}{2} e^{-|k| t} \int_{1}^{t}\left(h_{1,1}(k, s-1) \hat{Q}_{1}(k, s)+2 \sqrt{-i k}(s-1) \hat{Q}_{1}(0, s)\right) d s\right| \\
& \leq \text { const. } e^{-|k| t} \int_{1}^{t}\left|h_{1,1}(k, \sigma)+2 \sqrt{-i k} \sigma\right| \mu_{1}(k, s) d s \\
& + \text { const. } e^{-|k| t}|k|^{3 / 2} \int_{1}^{t}(s-1)\left|\partial_{k} \hat{Q}_{1}(\zeta, s)\right| d s .
\end{aligned}
$$

By (109) and (115) the second term is in $\mathcal{B}_{\alpha, \frac{3}{2}-\delta, \infty}$. Using (36) and Propositions 22 and 23 we also show that

$$
\begin{aligned}
& \left|e^{-|k| t} \int_{1}^{t}\left(h_{1,1}(k, s-1)+2 \sqrt{-i k}(s-1)\right) \hat{Q}_{1}(k, s) d s\right| \\
& \leq \text { const. }\left(\frac{1}{t} \bar{\mu}_{\alpha}(k, t)+\frac{1}{t^{5 / 2}} \bar{\mu}_{\alpha}(k, t)+\frac{1}{t^{3}} \tilde{\mu}_{\alpha}(k, t)\right),
\end{aligned}
$$

such that, all in all, $\hat{\psi}_{2}^{r, 1} \in \mathcal{B}_{\alpha, 1, \infty}$.

Finally, using (115), we have

$$
\left|\hat{\psi}_{3}^{r, 1}\right|=\left|e^{-|k| t} \int_{t}^{\infty} \sqrt{-i k}(s-1) \hat{Q}_{1}(0, s) d s\right| \leq \text { const. } e^{-|k| t}|k|^{1 / 2} \frac{1}{t^{3 / 2}} \in \mathcal{B}_{\alpha, 2, \infty} .
$$

Gathering the bounds on the $\hat{\psi}_{i}^{r, 1}$ yields (32), and by the opening remark of the proof also (33).

\subsection{Leading order in $\hat{\eta}$ and $\hat{\omega}$}

In view of Proposition 8 and Remark 9 , the leading order term of $\hat{\eta}$ and $\hat{\omega}$ are to be extracted from $\hat{\eta}_{1,1}$ and $\hat{\omega}_{1,1}$, respectively. We have (see [9]),

$$
\begin{aligned}
& \hat{\eta}_{1,1}(k, t)=\frac{1}{2} e^{-\kappa(t-1)} \int_{1}^{t} g_{1,1}(k, s-1) \hat{Q}_{1}(k, s) d s, \\
& \hat{\omega}_{1,1}(k, t)=\frac{1}{2} e^{-\kappa(t-1)} \int_{1}^{t} f_{1,1}(k, s-1) \hat{Q}_{1}(k, s) d s,
\end{aligned}
$$

with

$$
\begin{aligned}
g_{1,1}(k, \sigma) & =\frac{\kappa}{i k}\left(e^{\kappa \sigma}+\frac{(|k|+\kappa)^{2}}{i k} e^{-\kappa \sigma}-2 \frac{|k|(|k|+\kappa)}{i k} e^{-|k| \sigma}\right), \\
f_{1,1}(k, \sigma) & =\frac{i k}{\kappa} g_{1,1}(k, \sigma) .
\end{aligned}
$$

Formally, we get from (37) and (38)

$$
\begin{aligned}
& \lim _{t \rightarrow \infty} \hat{\eta}_{1,1}\left(k / t^{2}, t\right)=-c_{1} e^{-\sqrt{-i k}}=: \hat{\eta}_{1,1}^{1}(k), \\
& \lim _{t \rightarrow \infty} t \hat{\omega}_{1,1}\left(k / t^{2}, t\right)=c_{1} \sqrt{-i k} e^{-\sqrt{-i k}}=: \hat{\omega}_{1,1}^{1}(k),
\end{aligned}
$$

with $c_{1}$ as defined in (29). This motivates the definition of the functions

$$
\begin{aligned}
& \hat{\eta}_{\mathrm{as}, 1}(k, t)=\hat{\eta}_{1,1}^{1}\left(k t^{2}\right)=-c_{1} e^{-\sqrt{-i k} t} \\
& \hat{\omega}_{\mathrm{as}, 1}(k, t)=\frac{1}{t} \hat{\omega}_{1,1}^{1}\left(k t^{2}\right)=c_{1} \sqrt{-i k} e^{-\sqrt{-i k} t} .
\end{aligned}
$$


Note that $\hat{\eta}_{\mathrm{as}, 1} \in \mathcal{B}_{\alpha, \infty, 0}$ and $\hat{\omega}_{\mathrm{as}, 1} \in \mathcal{B}_{\alpha, \infty, 1}$. We now show that

$$
\begin{gathered}
\hat{\eta}_{1,1}-\hat{\eta}_{\mathrm{as}, 1} \in \mathcal{B}_{\alpha^{\prime}, \frac{3}{2}, 1}, \\
\hat{\omega}_{1,1}-\hat{\omega}_{\mathrm{as}, 1} \in \mathcal{B}_{\alpha^{\prime}, \frac{5}{2}, 2} .
\end{gathered}
$$

Proof. We have

with, see Appendix A.2.

$$
\hat{\omega}_{1,1}=\frac{i k}{\kappa} \hat{\eta}_{1,1}
$$

$$
\text { const. } \leq\left|\frac{i k}{\kappa}\right| \leq \text { const. } \min \left\{1,\left|\Lambda_{-}\right|\right\},
$$

which means that the bounds on $\hat{\omega}_{1,1}$ are the same as those for $\hat{\eta}_{1,1}$ for $|k|>1$, but have an additional factor of $\left|\Lambda_{-}\right|$for $|k| \leq 1$. This results in an increase of 1 in both the indices $p$ and $q$ for the components $\omega$ when compared to the ones for $\hat{\eta}$. This means that $\hat{\omega}$ decays $1 / t$ faster than $\hat{\eta}$, and since

$$
\lim _{t \rightarrow \infty} t \cdot \frac{i k}{t^{2} \kappa\left(k / t^{2}\right)}=-\sqrt{-i k},
$$

the asymptote of $\hat{\omega}$ is naturally derived from the one of $\hat{\eta}$. We therefore only present the details of the proof for $\hat{\eta}$, since the proof for $\omega$ can easily be recovered by inserting the appropriate factors in the proof for $\hat{\eta}$.

In order to prove (43) we set

$$
\hat{\eta}_{1,1}(k, t)-\hat{\eta}_{\mathrm{as}, 1}(k, t)=\sum_{i=1}^{4} \hat{\eta}_{i}^{r, 1},
$$

where

$$
\begin{aligned}
& \hat{\eta}_{1}^{r, 1}=\frac{1}{2}\left(e^{-\kappa(t-1)}-e^{-\kappa t}\right) \int_{1}^{t} g_{1,1}(k, s-1) \hat{Q}_{1}(k, s) d s \\
& \hat{\eta}_{2}^{r, 1}=\frac{1}{2} e^{-\kappa t} \int_{1}^{t}\left(g_{1,1}(k, s-1) \hat{Q}_{1}(k, s)+2(s-1) \hat{Q}_{1}(0, s)\right) d s \\
& \hat{\eta}_{3}^{r, 1}=-\left(e^{-\kappa t}-e^{-\sqrt{-i k t}}\right) \int_{1}^{t}(s-1) \hat{Q}_{1}(0, s) d s \\
& \hat{\eta}_{4}^{r, 1}=e^{-\sqrt{-i k t}} \int_{t}^{\infty}(s-1) \hat{Q}_{1}(0, s) d s .
\end{aligned}
$$

We have

$$
\left|g_{1,1}(k, \sigma)\right| \leq\left\{\begin{array}{ll}
\text { const. } \sigma e^{\left|\Lambda_{-}\right| \sigma} & \text { for }|k| \leq 1 \\
\text { const. }\left|\Lambda_{-}\right| e^{\left|\Lambda_{-}\right| \sigma} & \text { for }|k|>1
\end{array},\right.
$$

and we treat the two cases separately, using both times Propositions 19 and 20 For $|k| \leq 1$ we have

$$
\begin{aligned}
\left|\hat{\eta}_{1}^{r, 1}\right| & =\left|\frac{1}{2}\left(e^{-\kappa(t-1)}-e^{-\kappa t}\right) \int_{1}^{t} g_{1,1}(k, s-1) \hat{Q}_{1}(k, s) d s\right| \\
& \leq \text { const. } e^{\Lambda_{-}(t-1)}\left|\Lambda_{-}\right| \int_{1}^{t} \sigma e^{\left|\Lambda_{-}\right| \sigma} \mu_{1}(k, s) d s \\
& \leq \text { const. }\left(\frac{1}{t^{5 / 2}} \bar{\mu}_{\alpha}(k, t)+\frac{1}{t} \tilde{\mu}_{\alpha}(k, t)\right)
\end{aligned}
$$

and for $|k|>1$ we have, using (114),

$$
\begin{aligned}
\left|\hat{\eta}_{1}^{r, 1}\right| & =\left|\frac{1}{2}\left(e^{-\kappa(t-1)}-e^{-\kappa t}\right) \int_{1}^{t} g_{1,1}(k, s-1) \hat{Q}_{1}(k, s) d s\right| \\
& \leq \text { const. } e^{\Lambda_{-}(t-1)}\left|\Lambda_{-}\right| \int_{1}^{t}\left|\Lambda_{-}\right| e^{\left|\Lambda_{-}\right| \sigma} \mu_{1}(k, s) d s \\
& \leq \text { const. }\left(\frac{1}{t^{4}} \bar{\mu}_{\alpha-1}+\frac{1}{t^{3}} \tilde{\mu}_{\alpha-1}\right),
\end{aligned}
$$


so that $\hat{\eta}_{1}^{r, 1} \in \mathcal{B}_{\alpha^{\prime}, \frac{5}{2}, 1}$.

To bound $\hat{\eta}_{2}^{r, 1}$ we note that by (108)

$$
\begin{aligned}
& g_{1,1}(k, \sigma) \hat{Q}_{1}(k, s)+2 \sigma \hat{Q}_{1}(0, s) \\
& =\left(g_{1,1}(k, \sigma)+2 \sigma\right) \hat{Q}_{1}(k, s)-2 \sigma k \partial_{k} \hat{Q}_{1}(\zeta, s),
\end{aligned}
$$

for some $\zeta \in[0, k]$. We first analyze the expression

$$
g_{1,1}(k, \sigma)+2 \sigma=\frac{\kappa}{i k}\left(e^{\kappa \sigma}+\frac{(|k|+\kappa)^{2}}{i k} e^{-\kappa \sigma}-2 \frac{|k|(|k|+\kappa)}{i k} e^{-|k| \sigma}+2 \frac{i k}{\kappa} \sigma\right) .
$$

A straightforward bound is

$$
\left|g_{1,1}(k, \sigma)+2 \sigma\right| \leq\left\{\begin{array}{ll}
\text { const. } \sigma e^{\left|\Lambda_{-}\right| \sigma} & \text { for }|k| \leq 1 \\
\text { const. }\left(\sigma+1+\left|\Lambda_{-}\right|\right) e^{\left|\Lambda_{-}\right| \sigma} & \text { for }|k|>1
\end{array} .\right.
$$

Since the leading terms cancel, we also have

$$
\begin{aligned}
g_{1,1}(k, \sigma)+2 \sigma & =\frac{\kappa}{i k}\left(\left(e^{\kappa \sigma}-1-\kappa \sigma\right)-\left(e^{-\kappa \sigma}-1+\kappa \sigma\right)\right) \\
& +\frac{\kappa}{i k}\left(\frac{2|k|^{2}+2|k| \kappa}{i k}\left(\left(e^{-\kappa \sigma}-1\right)-\left(e^{-|k| \sigma}-1\right)\right)+2 \kappa \sigma+2 \frac{i k}{\kappa} \sigma\right),
\end{aligned}
$$

which we can bound by

$$
\begin{aligned}
\left|g_{1,1}(k, \sigma)+2 \sigma\right| & \leq \text { const. }\left|\frac{\kappa}{i k}\right|\left(\left|\Lambda_{-}\right|^{2} \sigma^{2} e^{\left|\Lambda_{-}\right| \sigma}+\left|\Lambda_{-}\right|^{2} \sigma^{2}+\left|\frac{2|k|^{2}+2|k| \kappa}{i k}\right|\left(\left|\Lambda_{-}\right| \sigma+|k| \sigma\right)+2\left|\Lambda_{-}\right|^{2} \sigma\right) \\
& \leq \begin{cases}\text { const. }\left|\Lambda_{-}\right| \sigma(\sigma+1) e^{\left|\Lambda_{-}\right| \sigma} & \text { for }|k| \leq 1 \\
\text { const. }\left|\Lambda_{-}\right|^{2} \sigma(\sigma+1) e^{\left|\Lambda_{-}\right| \sigma} & \text { for }|k|>1\end{cases}
\end{aligned}
$$

using that

$$
\left|\kappa+\frac{i k}{\kappa}\right| \leq\left|\frac{k^{2}-i k}{\kappa}+\frac{i k}{\kappa}\right| \leq \text { const. }|k|^{3 / 2} \leq \text { const. }\left|\Lambda_{-}\right|^{2} .
$$

Therefore, using (45) and (46), we get

$$
\left|g_{1,1}(k, \sigma)+2 \sigma\right| \leq \begin{cases}\text { const. } \sigma e^{\left|\Lambda_{-}\right| \sigma} \min \left\{1,\left|\Lambda_{-}\right|(\sigma+1)\right\} & \text { for }|k| \leq 1 \\ \text { const. } e^{\left|\Lambda_{-}\right| \sigma} \min \left\{\left(\sigma+1+\left|\Lambda_{-}\right|\right),\left|\Lambda_{-}\right|^{2} \sigma(\sigma+1)\right\} & \text { for }|k|>1\end{cases}
$$

Collecting these bounds yields

$$
\begin{aligned}
\left|\hat{\eta}_{2}^{r, 1}\right| & =\left|\frac{1}{2} e^{-\kappa t} \int_{1}^{t}\left(g_{1,1}(k, \sigma) \hat{Q}_{1}(k, s)+2 \sigma \hat{Q}_{1}(0, s)\right) d s\right| \\
& \leq \text { const. } e^{\Lambda_{-} t} \int_{1}^{t}\left|g_{1,1}(k, \sigma)+2 \sigma\right| \mu_{1}(k, s) d s+\text { const. } e^{\Lambda_{-} t}|k| \int_{1}^{t} \sigma\left|\partial_{k} \hat{Q}_{1}(\zeta, s)\right| d s .
\end{aligned}
$$

The second term of this inequality on $\left|\hat{\eta}_{2}^{r, 1}\right|$ can be integrated and bounded due to (109), and is in $\mathcal{B}_{\alpha, \infty, \frac{3}{2}}$ by (116). For the first term, using Propositions 19 and 20 with the bound (47) we have, for $|k| \leq 1$,

$$
\begin{aligned}
& \left|\frac{1}{2} e^{-\kappa(t-1)} \int_{1}^{t}\left(g_{1,1}(k, \sigma)+2 \sigma\right) \hat{Q}_{1}(k, s) d s\right| \\
& \leq \text { const. }\left(\frac{1}{t} \tilde{\mu}_{\alpha}(k, t)+\frac{1}{t^{3 / 2}} \bar{\mu}_{\alpha}(k, t)+\frac{1}{t^{2}} \tilde{\mu}_{\alpha}(k, t)\right),
\end{aligned}
$$

and for $|k|>1$,

$$
\begin{aligned}
& \left|\frac{1}{2} e^{-\kappa(t-1)} \int_{1}^{t}\left(g_{1,1}(k, \sigma)+2 \sigma\right) \hat{Q}_{1}(k, s) d s\right| \\
& \leq \text { const. }\left(\frac{1}{t^{2}} \tilde{\mu}_{\alpha}(k, t)+\frac{1}{t^{3 / 2}} \bar{\mu}_{\alpha}(k, t)+\frac{1}{t^{2}} \tilde{\mu}_{\alpha}(k, t)\right),
\end{aligned}
$$


which shows that $\hat{\eta}_{2}^{r, 1} \in \mathcal{B}_{\alpha, \frac{3}{2}, 1}$.

We now bound $\hat{\eta}_{3}^{r, 1}$, which, using (116), yields

$$
\left|\hat{\eta}_{3}^{r, 1}\right|=\left|\left(e^{-\kappa t}-e^{-\sqrt{-i k} t}\right) \int_{1}^{t}(s-1) \hat{Q}_{1}(0, s) d s\right| \leq \text { const. }\left|e^{-\sqrt{-i k} t}\right||k|^{3 / 2} t \in \mathcal{B}_{\alpha, \infty, 2} .
$$

Finally, using (116), we have

$$
\left|\hat{\eta}_{4}^{r, 1}\right|=\left|e^{-\sqrt{-i k} t} \int_{t}^{\infty}(s-1) \hat{Q}_{1}(0, s) d s\right| \leq \text { const. }\left|e^{-\sqrt{-i k} t}\right| \frac{1}{t^{3 / 2}} \in \mathcal{B}_{\alpha, \infty, \frac{3}{2}} .
$$

Gathering the bounds on the $\hat{\eta}_{i}^{r, 1}$ yields (43), and by the opening remark of the proof also (44).

\subsection{Leading order in $\partial_{k} \hat{\omega}$}

For technical reasons that will become clear in the procedure of extracting second order asymptotic terms, it is necessary to give tighter bounds on $\partial_{k} \hat{Q}_{1}=\hat{v} * \partial_{k} \hat{\omega}+\partial_{k} \hat{F}_{1}$ and $\partial_{k} \hat{\omega}$ (we recall that $\hat{\omega}$ is continuous on $\mathbb{R}$ and $C^{1}$ on $\mathbb{R} \backslash\{0\}$, and that the derivative on $\mathbb{R}$ is to be understood in the sense of distributions). From [1] we have

$$
\begin{aligned}
& \kappa \partial_{k} \hat{\omega} \in \mathcal{B}_{\alpha^{\prime}, \frac{3}{2}, 0}, \\
& \kappa \partial_{k} \hat{\omega}-\kappa \partial_{k} \hat{\omega}_{1,1,1}-\kappa \partial_{k} \hat{\omega}_{2,1,1} \in \mathcal{B}_{\alpha^{\prime}, \frac{3}{2}, 1},
\end{aligned}
$$

with

$$
\begin{aligned}
& \partial_{k} \hat{\omega}_{1,1,1}(k, t)=\frac{1}{2}\left(\partial_{k} e^{-\kappa \tau}\right) \int_{1}^{t} f_{1,1}(k, s-1) \hat{Q}_{1}(k, s) d s, \\
& \partial_{k} \hat{\omega}_{2,1,1}(k, t)=\frac{1}{2} e^{-\kappa \tau} \int_{1}^{t}\left(\partial_{k} f_{1,1}(k, s-1)\right) \hat{Q}_{1}(k, s) d s,
\end{aligned}
$$

with $f_{1,1}$ given by (40), with

$$
\partial_{k} \kappa=\frac{2 k-i}{2 \kappa},
$$

and

$$
\begin{aligned}
\partial_{k} f_{1,1}(k, \sigma) & =i \frac{(|k|+\kappa)^{2}}{\kappa|k|}\left(e^{-|k| \sigma}-e^{-\kappa \sigma}\right) \\
& +\frac{k^{2}+\kappa^{2}}{2 \kappa k}\left(e^{\kappa \sigma}+e^{-\kappa \sigma}\right) \sigma \\
& +2 i \frac{k^{2}+|k| \kappa}{k^{2}}\left(\frac{k^{2}+\kappa^{2}}{2 \kappa} e^{-\kappa \sigma}-|k| e^{-|k| \sigma}\right) \sigma .
\end{aligned}
$$

We have, from (42),

$$
\partial_{k} \hat{\omega}_{\mathrm{as}}(k)=i \frac{c_{1}}{2}\left(1-\frac{1}{\sqrt{-i k t}}\right) t e^{-\sqrt{-i k} t},
$$

with $c_{1}$ as defined by (29). Note that $\partial_{k} \hat{\omega}_{\text {as }} \in \mathcal{B}_{\alpha, \infty, 0}$. We now show that

$$
\kappa \partial_{k} \hat{\omega}_{1,1,1}+\kappa \partial_{k} \hat{\omega}_{2,1,1}-\kappa \partial_{k} \hat{\omega}_{\mathrm{as}} \in \mathcal{B}_{\alpha^{\prime \prime}, \frac{3}{2}, 1} .
$$

Remark 11 Note that

$$
\mathcal{F}^{-1}\left[-i \partial_{k} \hat{\omega}_{\mathrm{as}}(k, y)\right]=x \omega_{W}(x, y)=x \mathcal{F}^{-1}\left[\hat{\omega}_{\mathrm{as}, 1}(k, y)\right] .
$$

Proof. In order to prove (53) we note that

$$
\begin{aligned}
& \kappa \partial_{k} \hat{\omega}_{1,1,1}(k, t)+\kappa \partial_{k} \hat{\omega}_{2,1,1}(k, t)-\kappa \partial_{k} \hat{\omega}_{\mathrm{as}}(k, t) \\
& =-\frac{2 k-i}{4} \tau e^{-\kappa \tau} \int_{1}^{t} f_{1,1}(k, s-1) \hat{Q}_{1}(k, s) d s \\
& +\frac{1}{2} e^{-\kappa \tau} \int_{1}^{t} \kappa \partial_{k} f_{1,1}(k, s-1) \hat{Q}_{1}(k, s) d s \\
& -i \frac{\kappa}{2}\left(1-\frac{1}{\sqrt{-i k} t}\right) t e^{-\sqrt{-i k} t} \int_{1}^{\infty}(s-1) \hat{Q}_{1}(0, s) d s .
\end{aligned}
$$


We rewrite this expression as a sum of terms which can easily be bounded. Namely,

$$
\kappa \partial_{k} \hat{\omega}_{1,1,1}(k, t)+\kappa \partial_{k} \hat{\omega}_{2,1,1}(k, t)-\kappa \partial_{k} \hat{\omega}_{\text {as }}(k, t)=\sum_{i=1}^{5} \kappa \partial_{k} \hat{\omega}_{i}^{r},
$$

with

$$
\begin{aligned}
& \kappa \partial_{k} \hat{\omega}_{1}^{r}=-\frac{2 k-i}{4} \tau\left(e^{-\kappa(t-1)}-e^{-\kappa t}\right) \int_{1}^{t} f_{1,1}(k, s-1) \hat{Q}_{1}(k, s) d s \\
& \kappa \partial_{k} \hat{\omega}_{2}^{r}=-\frac{2 k-i}{4} \tau e^{-\kappa t} \int_{1}^{t} f_{1,1}(k, s-1) \hat{Q}_{1}(k, s) d s-i \frac{\kappa}{2} t e^{-\sqrt{-i k} t} \int_{1}^{t}(s-1) \hat{Q}_{1}(0, s) d s \\
& \kappa \partial_{k} \hat{\omega}_{3}^{r}=\frac{1}{2}\left(e^{-\kappa(t-1)}-e^{-\kappa t}\right) \int_{1}^{t} \kappa \partial_{k} f_{1,1}(k, s-1) \hat{Q}_{1}(k, s) d s \\
& \kappa \partial_{k} \hat{\omega}_{4}^{r}=\frac{1}{2} e^{-\kappa t} \int_{1}^{t} \kappa \partial_{k} f_{1,1}(k, s-1) \hat{Q}_{1}(k, s) d s+\frac{i \kappa}{2 \sqrt{-i k}} e^{-\sqrt{-i k} t} \int_{1}^{t}(s-1) \hat{Q}_{1}(0, s) d s \\
& \kappa \partial_{k} \hat{\omega}_{5}^{r}=-i \frac{\kappa}{2}\left(1-\frac{1}{\sqrt{-i k} t}\right) t e^{-\sqrt{-i k} t} \int_{t}^{\infty}(s-1) \hat{Q}_{1}(0, s) d s .
\end{aligned}
$$

In the rest of this proof, we apply without mention (114) to eliminate spurious powers of $\left|\Lambda_{-}\right|$whenever the conditions of Propositions 19 and 20 require it.

First we have

$$
\begin{aligned}
\left|\kappa \partial_{k} \hat{\omega}_{1}^{r}\right| & =\left|\frac{2 k-i}{4} \tau\left(e^{-\kappa(t-1)}-e^{-\kappa t}\right) \int_{1}^{t} f_{1,1}(k, s-1) \hat{Q}_{1}(k, s) d s\right| \\
& \leq \text { const. }(1+|k|) t e^{\Lambda_{-}(t-1)}\left|\Lambda_{-}\right| \int_{1}^{t}\left(1+\left|\Lambda_{-}\right|\right) e^{\left|\Lambda_{-}\right| \sigma} \min \left\{1,\left|\Lambda_{-}\right| \sigma\right\} \mu_{1}(k, s) d s \\
& \leq \text { const. } t\left(\frac{1}{t^{2}} \tilde{\mu}_{\alpha-2}+\frac{1}{t^{7 / 2}} \bar{\mu}_{\alpha-2}+\frac{1}{t^{4}} \tilde{\mu}_{\alpha-2}\right),
\end{aligned}
$$

showing that $\kappa \partial_{k} \hat{\omega}_{1}^{r} \in \mathcal{B}_{\alpha^{\prime \prime}, \frac{5}{2}, 1}$.

For $\kappa \partial_{k} \hat{\omega}_{2}^{r}$ we have

$$
\begin{aligned}
& -\frac{2 k-i}{4} \tau e^{-\kappa t} \int_{1}^{t} f_{1,1}(k, s-1) \hat{Q}_{1}(k, s) d s-i \frac{\kappa}{2} t e^{-\sqrt{-i k} t} \int_{1}^{t}(s-1) \hat{Q}_{1}(0, s) d s \\
& =-\frac{2 k-i}{4} t e^{-\kappa t} \int_{1}^{t} f_{1,1}(k, s-1) \hat{Q}_{1}(k, s) d s-i \frac{\kappa}{2} t e^{-\sqrt{-i k} t} \int_{1}^{t}(s-1) \hat{Q}_{1}(0, s) d s \\
& +e^{-\kappa t} \int_{1}^{t} \frac{2 k-i}{4} f_{1,1}(k, s-1) \hat{Q}_{1}(k, s) d s,
\end{aligned}
$$

where the last term can be bounded by applying Propositions 19 and 20, so that

$$
\begin{aligned}
& \left|e^{-\kappa t} \int_{1}^{t} \frac{2 k-i}{4} f_{1,1}(k, s-1) \hat{Q}_{1}(k, s) d s\right| \\
& \leq \text { const. } e^{\Lambda_{-} \tau} \int_{1}^{t}\left(1+\left|\Lambda_{-}\right|^{2}\right) e^{\left|\Lambda_{-}\right| \sigma} \min \left\{1,\left|\Lambda_{-}\right| \sigma\right\} \mu_{1}(k, s) d s \\
& \leq \text { const. }\left(\frac{1}{t} \tilde{\mu}_{\alpha-1}(k, t)+\frac{1}{t^{5 / 2}} \bar{\mu}_{\alpha-1}(k, t)+\frac{1}{t^{3}} \tilde{\mu}_{\alpha-1}(k, t)\right),
\end{aligned}
$$

whereas for (년), we get

$$
\begin{aligned}
& -\frac{2 k-i}{4} t e^{-\kappa t} \int_{1}^{t} f_{1,1}(k, s-1) \hat{Q}_{1}(k, s) d s-i \frac{\kappa}{2} t e^{-\sqrt{-i k} t} \int_{1}^{t}(s-1) \hat{Q}_{1}(0, s) d s \\
& =-\frac{t}{2}\left(e^{-\kappa t} \int_{1}^{t} \frac{2 k-i}{2} f_{1,1}(k, s-1) \hat{Q}_{1}(k, s) d s+e^{-\sqrt{-i k} t} \int_{1}^{t} i \kappa(s-1) \hat{Q}_{1}(0, s) d s\right) \\
& =\frac{t}{2}\left(e^{-\kappa t}-e^{-\sqrt{-i k} t}\right) \int_{1}^{t} i \kappa(s-1) \hat{Q}_{1}(0, s) d s \\
& -\frac{t}{2} e^{-\kappa t} \int_{1}^{t}\left(\frac{2 k-i}{2} f_{1,1}(k, s-1) \hat{Q}_{1}(k, s)+i \kappa(s-1) \hat{Q}_{1}(0, s)\right) d s .
\end{aligned}
$$


For (55) we get, using (111) and (116),

$$
\left|\frac{t}{2}\left(e^{-\kappa t}-e^{-\sqrt{-i k} t}\right) \int_{1}^{t} i \kappa(s-1) \hat{Q}_{1}(0, s) d s\right| \leq \text { const. } t\left|e^{-\sqrt{-i k} t}\right||k|^{3 / 2} t\left(|k|^{1 / 2}+|k|\right) \in \mathcal{B}_{\alpha, \infty, 2} .
$$

To bound (56) we note that, using (108),

$$
\begin{aligned}
& \frac{2 k-i}{2} f_{1,1}(k, s-1) \hat{Q}_{1}(k, s)+i \kappa(s-1) \hat{Q}_{1}(0, s) \\
& =\left(\frac{2 k-i}{2} f_{1,1}(k, s-1)+i \kappa(s-1)\right) \hat{Q}_{1}(k, s)+i \kappa k(s-1) \partial_{k} \hat{Q}_{1}(\zeta, s),
\end{aligned}
$$

for some $\zeta \in[0, k]$, which allows us to rewrite (56) as

$$
\begin{aligned}
& e^{-\kappa t} \int_{1}^{t}\left(\frac{2 k-i}{2} f_{1,1}(k, s-1) \hat{Q}_{1}(k, s)+i \kappa(s-1) \hat{Q}_{1}(0, s)\right) d s \\
& =e^{-\kappa t} \int_{1}^{t}\left(\frac{2 k-i}{2} f_{1,1}(k, s-1)+i \kappa(s-1)\right) \hat{Q}_{1}(k, s) d s \\
& +e^{-\kappa t} \int_{1}^{t} i \kappa k(s-1) \partial_{k} \hat{Q}_{1}(\zeta, s) d s .
\end{aligned}
$$

For the last term we have, using (116),

$$
\left|e^{-\kappa t} \int_{1}^{t} i \kappa k(s-1) \partial_{k} \hat{Q}_{1}(\zeta, s) d s\right| \leq \text { const. } e^{\Lambda_{-} t}|k|\left(|k|^{1 / 2}+|k|\right) \sqrt{t} \in \mathcal{B}_{\alpha, \infty, \frac{5}{2}} .
$$

To bound (57) we use that

$$
\begin{aligned}
\frac{2 k-i}{2} f_{1,1}(k, \sigma)+i \kappa \sigma & =\frac{2 k-i}{2}\left(e^{\kappa \sigma}+\frac{(|k|+\kappa)^{2}}{i k} e^{-\kappa \sigma}-2 \frac{|k|(|k|+\kappa)}{i k} e^{-|k| \sigma}\right)+i \kappa \sigma \\
& =k\left(e^{\kappa \sigma}+\frac{(|k|+\kappa)^{2}}{i k} e^{-\kappa \sigma}-2 \frac{|k|(|k|+\kappa)}{i k} e^{-|k| \sigma}\right) \\
& -\frac{i}{2}\left(\left(e^{\kappa \sigma}-1-\kappa \sigma\right)-\left(e^{-\kappa \sigma}-1+\kappa \sigma\right)\right) \\
& +\frac{|k|(|k|+\kappa)}{k}\left(\left(e^{-\kappa \sigma}-1\right)-\left(e^{-|k| \sigma}-1\right)\right),
\end{aligned}
$$

which, using the usual bound on $f_{1,1}$ and using the fact that leading order terms cancel where we put them in evidence, we get

$$
\begin{aligned}
& \left|\frac{2 k-i}{2} f_{1,1}(k, \sigma)+i \kappa \sigma\right| \\
& \leq \text { const. }\left(|k|\left(1+\left|\Lambda_{-}\right|\right) \min \left\{1,\left|\Lambda_{-}\right| \sigma\right\}+\min \left\{1,\left|\Lambda_{-}\right| \sigma\right\}\left|\Lambda_{-}\right| \sigma+\left|\Lambda_{-}\right|^{2} \sigma\right) e^{\left|\Lambda_{-}\right| \sigma},
\end{aligned}
$$

which, using Propositions 19 and 20, yields

$$
e^{-\kappa t} \int_{1}^{t}\left(\frac{2 k-i}{2} f_{1,1}(k, s-1)+i \kappa(s-1)\right) \hat{Q}_{1}(k, s) d s \in \mathcal{B}_{\alpha-1, \frac{5}{2}, 2} .
$$

All in all, we thus have $\kappa \partial_{k} \hat{\omega}_{2}^{r} \in \mathcal{B}_{\alpha^{\prime}, \frac{5}{2}, 1}$.

To bound $\kappa \partial_{k} \hat{\omega}_{3}^{r}$ we use the bound (see [1])

$$
\left|\kappa \partial_{k} f_{1,1}(k, \sigma)\right| \leq \text { const. }\left(1+\left|\Lambda_{-}\right|^{2}\right) \sigma e^{\left|\Lambda_{-}\right| \sigma},
$$

and using Propositions 19 and 20 we get

$$
\begin{aligned}
& \left|\frac{1}{2}\left(e^{-\kappa(t-1)}-e^{-\kappa t}\right) \int_{1}^{t} \kappa \partial_{k} f_{1,1}(k, s-1) \hat{Q}_{1}(k, s) d s\right| \\
& \leq \text { const. } e^{\Lambda_{-}(t-1)}\left|\Lambda_{-}\right| \int_{1}^{t}\left(1+\left|\Lambda_{-}\right|^{2}\right) \sigma e^{\left|\Lambda_{-}\right| \sigma} \mu_{1}(k, s) d s \\
& \leq \text { const. }\left(\frac{1}{t^{1}} \tilde{\mu}_{\alpha-1}+\frac{1}{t^{5 / 2}} \bar{\mu}_{\alpha-2}-\frac{1}{t^{3}} \bar{\mu}_{\alpha-2}\right) .
\end{aligned}
$$


Thus, $\kappa \partial_{k} \hat{\omega}_{3}^{r} \in \mathcal{B}_{\alpha^{\prime \prime}, \frac{5}{2}, 1}$.

To bound $\kappa \partial_{k} \hat{\omega}_{4}^{r}$ we use that

$$
\begin{aligned}
\kappa \partial_{k} \hat{\omega}_{4}^{r} & =\frac{1}{2} e^{-\kappa t} \int_{1}^{t} \kappa \partial_{k} f_{1,1}(k, s-1) \hat{Q}_{1}(k, s) d s-\frac{1}{2} e^{-\sqrt{-i k} t} \int_{1}^{t} \frac{\kappa \sqrt{-i k}}{k}(s-1) \hat{Q}_{1}(0, s) d s \\
& =\frac{1}{2}\left(e^{-\kappa t}-e^{-\sqrt{-i k t}}\right) \int_{1}^{t} \frac{\kappa \sqrt{-i k}}{k}(s-1) \hat{Q}_{1}(0, s) d s \\
& +\frac{1}{2} e^{-\kappa t} \int_{1}^{t}\left(\kappa \partial_{k} f_{1,1}(k, s-1) \hat{Q}_{1}(k, s)-\frac{\kappa \sqrt{-i k}}{k}(s-1) \hat{Q}_{1}(0, s)\right) d s .
\end{aligned}
$$

We first bound (58) using (111) and (116). We have

$$
\begin{aligned}
& \left|\frac{1}{2}\left(e^{-\kappa t}-e^{-\sqrt{-i k} t}\right) \int_{1}^{t} \frac{\kappa \sqrt{-i k}}{k}(s-1) \hat{Q}_{1}(0, s) d s\right| \\
& \leq \text { const. }\left|e^{-\sqrt{-i k} t}\right||k|^{3 / 2} t \frac{\left(|k|^{1 / 2}+|k|\right)|k|^{1 / 2}}{|k|} \in \mathcal{B}_{\alpha, \infty, 2} .
\end{aligned}
$$

To bound (59) we note that, using (108),

$$
\begin{aligned}
& \kappa \partial_{k} f_{1,1}(k, s-1) \hat{Q}_{1}(k, s)-\frac{\kappa \sqrt{-i k}}{k}(s-1) \hat{Q}_{1}(0, s) \\
& =\left(\kappa \partial_{k} f_{1,1}(k, s-1)-\frac{\kappa \sqrt{-i k}}{k}(s-1)\right) \hat{Q}_{1}(k, s)+\frac{\kappa \sqrt{-i k}}{k} k(s-1) \partial_{k} \hat{Q}_{1}(\zeta, s),
\end{aligned}
$$

for some $\zeta \in[0, k]$. We next analyze

$$
\begin{aligned}
\kappa \partial_{k} f_{1,1}(k, \sigma)-\frac{\kappa \sqrt{-i k}}{k} \sigma & =i \frac{(|k|+\kappa)^{2}}{|k|}\left(\left(e^{-|k| \sigma}-1\right)-\left(e^{-\kappa \sigma}-1\right)\right) \\
& +\frac{k^{2}+\kappa^{2}}{2 k}\left(\left(e^{\kappa \sigma}-1\right)+\left(e^{-\kappa \sigma}-1\right)\right) \sigma \\
& +2 i \frac{k^{2}+|k| \kappa}{k^{2}}\left(\frac{k^{2}+\kappa^{2}}{2} e^{-\kappa \sigma}-|k| \kappa e^{-|k| \sigma}\right) \sigma \\
& +\frac{k^{2}+\kappa^{2}}{k} \sigma-\frac{\kappa \sqrt{-i k}}{k} \sigma .
\end{aligned}
$$

For the last line we have, using (110),

$$
\begin{aligned}
\left|\frac{k^{2}+\kappa^{2}}{k}-\frac{\kappa \sqrt{-i k}}{k}\right| & \leq|k|+\text { const. } \frac{|\nsupseteq-|}{|k|} \min \left\{\left|\Lambda_{-}\right|^{2},\left|\Lambda_{-}\right|^{3}\right\} \\
& \leq \text { const. }\left(|k|+|k|^{3}\right) \leq \text { const. }\left|\Lambda_{-}\right|^{2}\left(1+\left|\Lambda_{-}\right|\right),
\end{aligned}
$$

and therefore

$$
\left|\kappa \partial_{k} f_{1,1}(k, \sigma)-\frac{\kappa \sqrt{-i k}}{k} \sigma\right| \leq \text { const. }\left(1+\left|\Lambda_{-}\right|^{2}\right)\left|\Lambda_{-}\right| \sigma(\sigma+1) e^{\left|\Lambda_{-}\right| \sigma} .
$$

We can now bound (59). Namely, we have,

$$
\begin{aligned}
& \left|\frac{1}{2} e^{-\kappa t} \int_{1}^{t}\left(\kappa \partial_{k} f_{1,1}(k, s-1) \hat{Q}_{1}(k, s)-\frac{\kappa \sqrt{-i k}}{k}(s-1) \hat{Q}_{1}(0, s)\right) d s\right| \\
& =\left|\frac{1}{2} e^{-\kappa t} \int_{1}^{t}\left(\kappa \partial_{k} f_{1,1}(k, s-1)-\frac{\kappa \sqrt{-i k}}{k}(s-1)\right) \hat{Q}_{1}(k, s) d s\right| \\
& +\left|\frac{1}{2} e^{-\kappa t} \int_{1}^{t} \kappa \sqrt{-i k}(s-1) \partial_{k} \hat{Q}_{1}(\zeta, s) d s\right| \\
& \leq \text { const. } e^{\Lambda_{-} t} \int_{1}^{t}\left(1+\left|\Lambda_{-}\right|^{2}\right)\left|\Lambda_{-}\right| \sigma s e^{\left|\Lambda_{-}\right| \sigma} \mu_{1}(k, s) d s \\
& + \text { const. } e^{\Lambda_{-} t}\left(|k|^{1 / 2}+|k|\right)|k|^{1 / 2} \sqrt{t}
\end{aligned}
$$


where by Propositions 19 and 20, and inequality (114), the first term is in $\mathcal{B}_{\alpha-2, \frac{3}{2}, 1}$ and where due to (116) the second term is in $\mathcal{B}_{\alpha, \infty, \frac{3}{2}}$. Thus we get $\kappa \partial_{k} \hat{\omega}_{4}^{r} \in \mathcal{B}_{\alpha^{\prime \prime}, \frac{3}{2}, 1}$.

Finally, to bound $\kappa \partial_{k} \hat{\omega}_{5}^{r}$, we use (116), so that

$$
\begin{aligned}
& \left|i \frac{\kappa}{2}\left(1-\frac{1}{\sqrt{-i k} t}\right) t e^{-\sqrt{-i k} t} \int_{t}^{\infty}(s-1) \hat{Q}_{1}(0, s) d s\right| \\
& \leq \text { const. }\left(|k|^{1 / 2} t+1\right)\left(1+|k|^{1 / 2}\right)\left|e^{-\sqrt{-i k} t}\right| \frac{1}{t^{3 / 2}} \in \mathcal{B}_{\alpha, \infty, 3 / 2} .
\end{aligned}
$$

Gathering all the bounds on the $\kappa \partial_{k} \hat{\omega}_{i}^{r}$ leads to (53).

\subsection{Improvement of the bounds on the non-linear terms}

Improvement of the bounds on $\hat{Q}_{0}$ and $\hat{Q}_{1}$

From Section 3 we know that

$$
\hat{Q}_{1}=(\hat{\omega}+\hat{\psi}) * \hat{\omega}+\hat{F}_{2} \in \mathcal{B}_{\alpha, \frac{7}{2}, 4} .
$$

The force term $\hat{F}_{2}$ is a function of rapid decrease in $k$ and of compact support in $t$ and will thus not intervene in our bounds. Using (42), (44), (30), (32), Propositions 8 and 16 we have

$$
\begin{aligned}
\hat{\omega} * \hat{\omega} & \in \mathcal{B}_{\alpha, \frac{11}{2}, 4}, \\
\hat{\psi} *\left(\hat{\omega}-\hat{\omega}_{\mathrm{as}, 1}\right) & \in \mathcal{B}_{\alpha^{\prime}, 4, \infty}, \\
\left(\hat{\psi}-\hat{\psi}_{\mathrm{as}, 1}\right) * \hat{\omega}_{\mathrm{as}, 1} & \in \mathcal{B}_{\alpha^{\prime}, 4, \infty} .
\end{aligned}
$$

For the term $\hat{\psi}_{\mathrm{as}, 1} * \hat{\omega}_{\mathrm{as}, 1}$ we can take advantage of the particular form of the explicit functions in direct space in order to improve the index $p$ by $1 / 2$ in comparison to what would be possible with the bounds on the convolution. In direct space we have,

$$
Q_{1}^{\mathrm{d}}=\mathcal{F}^{-1}\left[\hat{Q}_{1}^{\mathrm{d}}\right]=\mathcal{F}^{-1}\left[\hat{\psi}_{\mathrm{as}, 1} * \hat{\omega}_{\mathrm{as}, 1}\right]=\frac{1}{y^{3 / 2}} \psi_{1}(x / y) \cdot \frac{1}{y^{3}} \omega_{W}\left(x / y^{2}\right),
$$

where $\psi_{1}$ and $\omega_{W}$ are explicitly represented by (98) and (105) in Appendix A.1 We use various properties of these functions as well as their derivatives of order $n$, represented by the superscript ${ }^{(n)}$, which are easily understood from their explicit representation and shall thus not be proved. We show that using the definition of the function spaces $\mathcal{B}_{\alpha, \infty, q}$ we can improve the bound on $\hat{Q}_{1}$. We require that all the terms of the form

$$
\left|\left(|k| y^{2}\right)^{a} \hat{Q}_{1}^{\mathrm{d}}(k, y)\right|=\left(|k| y^{2}\right)^{a}\left|\int_{\mathbb{R}} e^{i k x} \frac{1}{y^{9 / 2}} \psi_{1}(x / y) \omega_{W}\left(x / y^{2}\right) d x\right|,
$$

for $a \in \mathbb{N}, 0 \leq a \leq\lfloor\alpha\rfloor+1$, be bounded. Since, for $n \geq 0$, all the $\psi_{1}^{(n)}$ and $\omega_{W}^{(n)}$ are in $C^{\infty}(\mathbb{R})$ and vanish for $|z| \rightarrow \infty$, we may integrate by parts and we have

$$
\left|\left(|k| y^{2}\right)^{a} \hat{Q}_{1}^{\mathrm{d}}(k, y)\right|=y^{2 a}\left|\int_{\mathbb{R}} e^{i k x} \frac{1}{y^{9 / 2}} \partial_{x}^{a}\left(\psi_{1}(x / y) \omega_{W}\left(x / y^{2}\right)\right) d x\right| .
$$

We then make use of the Newton binomial to expand the partial derivative of a product of functions in terms of a product of ordinary derivatives,

$$
\begin{aligned}
& \left|\left(|k| y^{2}\right)^{a} \hat{Q}_{1}^{\mathrm{d}}(k, y)\right| \\
& \leq \text { const. } y^{2 a} \int_{\mathbb{R}} \frac{1}{y^{9 / 2}} \sum_{n=0}^{a}\left(\begin{array}{c}
a \\
n
\end{array}\right) \frac{1}{y^{n}}\left|\hat{\psi}_{1}^{(n)}(x / y)\right| \frac{1}{y^{2(a-n)}}\left|\omega_{W}^{(a-n)}\left(x / y^{2}\right)\right| d x .
\end{aligned}
$$

Using the essential fact that

$$
\sup _{z \in \mathbb{R}}\left\{|z|^{n+3 / 2}\left|\psi_{1}^{(n)}(z)\right|\right\}=\text { const. }<\infty, n \geq 0,
$$


and that all the $\omega_{W}^{(n)}$ are zero for $z<0$, we have

$$
\left|\left(|k| y^{2}\right)^{a} \hat{Q}_{1}^{\mathrm{d}}(k, y)\right| \leq \text { const. } \sum_{n=0}^{a} \frac{1}{y^{9 / 2-n}} \int_{0}^{\infty}\left|\frac{y^{n+3 / 2}}{x^{n+3 / 2}} \omega_{W}^{(a-n)}\left(x / y^{2}\right)\right| d x .
$$

Finally, using the change of variables $z=x / y^{2}$ and the crucial fact that all the $\omega_{W}^{(n)}$ have exponential decay when $z \rightarrow 0$, we have

$$
\begin{aligned}
\left|\left(|k| y^{2}\right)^{a} \hat{Q}_{1}^{\mathrm{d}}(k, y)\right| & \leq \text { const. } \sum_{n=0}^{a} \frac{y^{n+3 / 2}}{y^{9 / 2-n}} \int_{0}^{\infty}\left|\frac{\omega_{W}^{(a-n)}(z)}{y^{2 n+3} z^{n+3 / 2}}\right| y^{2} d z \\
& \leq \text { const. } \sum_{n=0}^{a} \frac{1}{y^{4}} \int_{0}^{\infty}\left|\frac{\omega_{W}^{(a-n)}(z)}{z^{n+3 / 2}}\right| d z \leq \text { const. } \frac{1}{y^{4}} .
\end{aligned}
$$

From this we have $q=4$ and thus $\hat{\psi}_{\mathrm{as}, 1} * \hat{\omega}_{\mathrm{as}, 1} \in \mathcal{B}_{\alpha, \infty, 4}$. We conclude, with (60)-(62), that

$$
\hat{Q}_{1} \in \mathcal{B}_{\alpha-1,4,4} \text {. }
$$

Similarly, we have

$$
\hat{Q}_{0} \in \mathcal{B}_{\alpha-1,4,3},
$$

where the index $q=3$ is due to the product $\hat{\omega} * \hat{\eta}$. In light of (64) we define

$$
\mu_{1}^{I}:=\frac{1}{s^{4}} \bar{\mu}_{\alpha^{\prime}}(k, s)+\frac{1}{s^{4}} \tilde{\mu}_{\alpha^{\prime}}(k, s),
$$

to replace (13) from now on.

\subsubsection{New bounds}

It is now possible to reevaluate the bounds on all functions presented in Proposition 8 .

Proposition 12 Let $\alpha^{\prime}>1$ and $\delta>0$. We have

$$
\begin{array}{llll}
\hat{\psi}_{1,0} \in \mathcal{B}_{\alpha^{\prime}, \frac{3}{2}-\delta, 2} & \hat{\psi}_{1,1} \in \mathcal{B}_{\alpha^{\prime}, \frac{1}{2}, 3} & \hat{\varphi}_{1,0} \in \mathcal{B}_{\alpha^{\prime}, \frac{3}{2}-\delta, 2} & \hat{\varphi}_{1,1} \in \mathcal{B}_{\alpha^{\prime}, \frac{1}{2}, 3} \\
\hat{\psi}_{2,0} \in \mathcal{B}_{\alpha^{\prime}, 3,2} & \hat{\psi}_{2,1} \in \mathcal{B}_{\alpha^{\prime}, 3,3} & \hat{\varphi}_{2,0} \in \mathcal{B}_{\alpha^{\prime}, 3,2} & \hat{\varphi}_{2,1} \in \mathcal{B}_{\alpha^{\prime}, 3,3} \\
\hat{\psi}_{3,0} \in \mathcal{B}_{\alpha^{\prime}, 3,2} & \hat{\psi}_{3,1} \in \mathcal{B}_{\alpha^{\prime}, 3,3} & \hat{\varphi}_{3,0} \in \mathcal{B}_{\alpha^{\prime}, 3,2} & \hat{\varphi}_{3,1} \in \mathcal{B}_{\alpha^{\prime}, 3,3} \\
\hat{\omega}_{1,0} \in \mathcal{B}_{\alpha^{\prime}, 4,3-\delta} & \hat{\omega}_{1,1} \in \mathcal{B}_{\alpha^{\prime}, 3,1} & \hat{\eta}_{1,0} \in \mathcal{B}_{\alpha^{\prime}, 3,2-\delta} & \hat{\eta}_{1,1} \in \mathcal{B}_{\alpha^{\prime}, 2,0} \\
\hat{\omega}_{2,0} \in \mathcal{B}_{\alpha^{\prime}, \infty, 3} & \hat{\omega}_{2,1} \in \mathcal{B}_{\alpha^{\prime}, \infty, 3} & \hat{\eta}_{2,0} \in \mathcal{B}_{\alpha^{\prime}, \infty, 2} & \hat{\eta}_{2,1} \in \mathcal{B}_{\alpha^{\prime}, \infty, 3} \\
\hat{\omega}_{3,0} \in \mathcal{B}_{\alpha^{\prime}, 4,3} & \hat{\omega}_{3,1} \in \mathcal{B}_{\alpha^{\prime}, 3,3} & \hat{\eta}_{3,0} \in \mathcal{B}_{\alpha^{\prime}, 3,2} & \hat{\eta}_{3,1} \in \mathcal{B}_{\alpha^{\prime}, 2,2}
\end{array}
$$

Proof. This is straightforward by the new bounds (65) and (64). For $\hat{\omega}_{2,1}$ and $\hat{\eta}_{2,1}$ we make use of an existing factor $e^{\Lambda_{-}(t-1)}$ (see [9]) and apply (113), just as was done for $\hat{\omega}_{2,0}$ and $\hat{\eta}_{2,0}$ in the proof of Proposition 8

Remark 13 We also have

$$
\begin{aligned}
\hat{\omega}-\hat{\omega}_{\mathrm{as}, 1} & \in \mathcal{B}_{\alpha^{\prime}, 3,2}, \\
\hat{\eta}-\hat{\eta}_{\mathrm{as}, 1} & \in \mathcal{B}_{\alpha^{\prime}, 2,1} .
\end{aligned}
$$

\section{Improvement of the bound on $\partial_{k} \hat{Q}_{1}$}

From [1] we have

$$
\partial_{k} \hat{Q}_{1}=\hat{v} * \partial_{k} \hat{\omega}+\partial_{k} \hat{F}_{2} \in \mathcal{B}_{\alpha, \frac{3}{2}, 2} .
$$

The term $\partial_{k} \hat{F}_{2}$ is a function of rapid decrease in $k$ and of compact support in $t$ and will thus not intervene in our bounds We use Propositions 12 and 18, (32), (48) and (53) to show that

$$
\begin{aligned}
\hat{\omega} * \partial_{k} \hat{\omega} & \in \mathcal{B}_{\alpha^{\prime}, 4,2}, \\
\hat{\psi} *\left(\partial_{k} \hat{\omega}-\partial_{k} \hat{\omega}_{\mathrm{as}, 1}\right) & \in \mathcal{B}_{\alpha^{\prime \prime}, \frac{5}{2}, \infty}, \\
\left(\hat{\psi}-\hat{\psi}_{\mathrm{as}, 1}\right) * \partial_{k} \hat{\omega}_{\mathrm{as}, 1} & \in \mathcal{B}_{\alpha^{\prime}, 2, \infty} .
\end{aligned}
$$


Since

$$
\mathcal{F}^{-1}\left[\partial_{k} \hat{\omega}_{\text {as }}\right](x, y)=\frac{x}{y^{3}} \omega_{W}\left(x / y^{2}\right)
$$

we again use property (63) of $\psi_{\mathrm{as}, 1}$ and the fact that $\omega_{\mathrm{as}, 1}^{(n)}(z<0)=0$, for all $n$, to show that the convolution product $\hat{\psi}_{\text {as }, 1} * \partial_{k} \hat{\omega}_{\text {as }}$ can be bounded in direct space in order to improve the index $p$ by $1 / 2$ in comparison to what would be possible with the bounds on convolution. The calculation is slightly longer than in the previous section, but the steps are exactly the same, so that we omit the details of the proof for the sake of concision. We finally have

$$
\hat{\psi}_{\mathrm{as}, 1} * \partial_{k} \hat{\omega}_{\mathrm{as}} \in \mathcal{B}_{\alpha, \infty, 2},
$$

and we thus get

$$
\partial_{k} \hat{Q}_{1} \in \mathcal{B}_{\alpha^{\prime \prime}, 2,2}
$$

\subsection{Second order in $\hat{\psi}$ and $\hat{\varphi}$}

Applying the new bound (64) for $\hat{Q}_{1}$ in a straightforward manner, and in view of Proposition 12 and Remark 9, we find that the second order terms of $\hat{\psi}$ and $\hat{\varphi}$ are to be extracted from $\hat{\psi}_{1,1}-\hat{\psi}_{\text {as }, 1}$ and $\hat{\varphi}_{1,1}-\hat{\varphi}_{\text {as }, 1}$, respectively. Inspecting the limits of these quantities motivates us, in a similar way as in the case of the leading order of $\hat{\psi}$ and $\hat{\varphi}$, to define the functions

$$
\begin{aligned}
& \hat{\psi}_{\mathrm{as}, 2}(k, t)=-\left(c_{1}|k|+\frac{1}{2} c_{2} i k\right) e^{-|k| t}, \\
& \hat{\varphi}_{\mathrm{as}, 2}(k, t)=-\left(c_{1} i k-\frac{1}{2} c_{2}|k|\right) e^{-|k| t},
\end{aligned}
$$

with $c_{1}$ as defined by (29) and

$$
c_{2}=\int_{1}^{\infty}(s-1)^{2} \hat{Q}_{1}(0, s) d s .
$$

Note that $\hat{\psi}_{\mathrm{as}, 2}, \hat{\varphi}_{\mathrm{as}, 2} \in \mathcal{B}_{\alpha^{\prime}, 1, \infty}$. We now show that

$$
\begin{aligned}
& \hat{\psi}_{1,1}-\hat{\psi}_{\mathrm{as}, 1}-\hat{\psi}_{\mathrm{as}, 2} \in \mathcal{B}_{\alpha^{\prime \prime}, \frac{3}{2}-\delta, \infty}, \\
& \hat{\varphi}_{1,1}-\hat{\varphi}_{\mathrm{as}, 1}-\hat{\varphi}_{\mathrm{as}, 2} \in \mathcal{B}_{\alpha^{\prime \prime}, \frac{3}{2}-\delta, \infty} .
\end{aligned}
$$

Proof. As already for the leading order term, we have

$$
\hat{\psi}_{1,1}-\hat{\psi}_{\mathrm{as}, 1}=\frac{|k|}{i k}\left(\hat{\varphi}_{1,1}-\hat{\varphi}_{\mathrm{as}, 1}\right)
$$

so that all bounds for $\hat{\psi}-\hat{\psi}_{\text {as, } 1}$ are the same as the ones for $\hat{\varphi}-\hat{\varphi}_{\text {as, } 1}$ and we only need to present the proof for $\hat{\psi}$. We set

$$
\hat{\psi}_{1,1}(k, t)-\hat{\psi}_{\mathrm{as}, 1}(\hat{\psi}, t)-\hat{\psi}_{\mathrm{as}, 2}(k, t)=\sum_{i=1}^{4} \hat{\psi}_{i}^{r, 2}
$$

where

$$
\begin{aligned}
& \hat{\psi}_{1}^{r, 2}=\frac{1}{2}\left(e^{-|k|(t-1)}-e^{-|k| t}\right) \int_{1}^{t}\left(h_{1,1}(k, \sigma)+2 \kappa \sigma\right) \hat{Q}_{1}(k, s) d s \\
& \hat{\psi}_{2}^{r, 2}=\frac{1}{2} e^{-|k| t} \int_{1}^{t}\left(\left(h_{1,1}(k, \sigma)+2 \kappa \sigma\right) \hat{Q}_{1}(k, s)+(2|k|+i k \sigma) \sigma \hat{Q}_{1}(0, s)\right) d s, \\
& \hat{\psi}_{3}^{r, 2}=e^{-|k| t} \int_{1}^{t} \sqrt{-i k} \sigma \hat{Q}_{1}(0, s) d s-e^{-|k|(t-1)} \int_{1}^{t} \kappa \sigma \hat{Q}_{1}(k, s) d s \\
& \hat{\psi}_{4}^{r, 2}=\frac{1}{2} e^{-|k| t} \int_{t}^{\infty}(2 \sqrt{-i k}+2|k|+i k \sigma) \sigma \hat{Q}_{1}(0, s) d s .
\end{aligned}
$$

We first derive some bounds on $h_{1,1}$, given by (27). One has the straightforward bound

$$
\left|h_{1,1}(k, \sigma)+2 \kappa \sigma\right| \leq \text { const. }\left(1+|k|+\left(|k|^{1 / 2}+|k|\right) \sigma\right) e^{|k| \sigma}
$$


and since the leading order terms cancel, we also have

$$
\begin{aligned}
& \left|h_{1,1}(k, \sigma)+2 \kappa \sigma\right| \\
& \leq\left|\left(1-e^{|k| \sigma}\right)+\frac{(|k|+\kappa)^{2}}{i k}\left(e^{-|k| \sigma}-1\right)-2 \frac{\kappa(|k|+\kappa)}{i k}\left(e^{-\kappa \sigma}-1\right)+2 \kappa \sigma\right| \\
& \leq\left|\left(1-e^{|k| \sigma}\right)+\frac{(|k|+\kappa)^{2}}{i k}\left(e^{-|k| \sigma}-1\right)-2 \frac{\kappa|k|+|k|^{2}}{i k}\left(e^{-\kappa \sigma}-1\right)+2\left(e^{-\kappa \sigma}-1\right)+2 \kappa \sigma\right| \\
& \leq \text { const. }\left(|k| \sigma+(1+|k|)|k| \sigma+\left(|k|^{1 / 2}+|k|\right)^{2} \sigma+\left(\left(|k|^{1 / 2}+|k|\right) \sigma\right)^{c+1}\right) e^{|k| \sigma} \\
& \leq \text { const. }\left(|k|^{(c+1) / 2}+|k|^{2}\right) \sigma(\sigma+1)^{c} e^{|k| \sigma},
\end{aligned}
$$

with $c=\{0,1\}$ depending on whether we use the $2 \kappa \sigma$ term to cancel an additional term in the last exponential or not. We have another straightforward bound, namely

$$
\left|h_{1,1}(k, \sigma)+2 \kappa \sigma+2\right| k\left|\sigma+i k \sigma^{2}\right| \leq \text { const. }\left(1+|k|+|k|^{1 / 2} \sigma+|k| \sigma(\sigma+1)\right) e^{|k| \sigma}
$$

and, using that leading order terms cancel, we also have

$$
\begin{aligned}
& \left|h_{1,1}(k, \sigma)+2 \kappa \sigma+2\right| k\left|\sigma+i k \sigma^{2}\right| \\
& \leq\left|-e^{|k| \sigma}-e^{-|k| \sigma}+2 \frac{|k|(|k|+\kappa)}{i k} e^{-|k| \sigma}-2 \frac{\kappa(|k|+\kappa)}{i k} e^{-\kappa \sigma}+2 \kappa \sigma+2\right| k\left|\sigma+i k \sigma^{2}\right| \\
& \leq\left.\left|-\left(e^{-|k| \sigma}-1+|k| \sigma-\frac{1}{2}|k|^{2} \sigma^{2}\right)-\left(e^{|k| \sigma}-1-|k| \sigma-\frac{1}{2}|k|^{2} \sigma^{2}\right)-\right| k\right|^{2} \sigma^{2} \\
& +2 \frac{|k|(|k|+\kappa)}{i k}\left(e^{-|k| \sigma}-1+|k| \sigma\right)-2 \frac{\kappa(|k|+\kappa)}{i k}\left(e^{-\kappa \sigma}-1+\kappa \sigma\right)+i k \sigma^{2} \mid .
\end{aligned}
$$

Rearranging the terms we get

$$
\begin{aligned}
& \left|h_{1,1}(k, \sigma)+2 \kappa \sigma+2\right| k\left|\sigma+i k \sigma^{2}\right| \\
& \leq \mid-\left(e^{-|k| \sigma}-1+|k| \sigma-\frac{1}{2}|k|^{2} \sigma^{2}\right)-\left(e^{|k| \sigma}-1-|k| \sigma-\frac{1}{2}|k|^{2} \sigma^{2}\right) \\
& +2 \frac{|k|(|k|+\kappa)}{i k}\left(e^{-|k| \sigma}-1+|k| \sigma\right)-2 \frac{|k|(|k|+\kappa)}{i k}\left(e^{-\kappa \sigma}-1+\kappa \sigma\right) \\
& +2\left(e^{-\kappa \sigma}-1+\kappa \sigma\right)-\kappa \sigma^{2} \mid \\
& \leq \text { const. }\left(\left(|k|^{3} \sigma^{3}\right)+\left(|k|^{1 / 2}+|k|\right)\left(|k|^{2}+|\kappa|^{2}\right) \sigma^{2}+\left(|\kappa|^{3} \sigma^{3}\right)\right) e^{|k| \sigma} \\
& \leq \text { const. }\left(|k|^{3 / 2}+|k|^{3}\right) \sigma^{2}(\sigma+1) .
\end{aligned}
$$

We now bound the terms $\hat{\psi}_{i}^{r, 2}$. Using Proposition 22 with the bound (78) and Proposition 23 as with (79), as well as inequality (114) where necessary, we have

$$
\begin{aligned}
\left|\hat{\psi}_{1}^{r, 2}\right| & =\left|\frac{1}{2}\left(e^{-|k|(t-1)}-e^{-|k| t}\right) \int_{1}^{t}\left(h_{1,1}(k, \sigma)+2 \kappa \sigma\right) \hat{Q}_{1}(k, s) d s\right| \\
& \leq \text { const. } e^{-|k| t}\left|e^{|k|}-1\right| \int_{1}^{t}\left|h_{1,1}(k, \sigma)+2 \kappa \sigma\right| \mu_{1}^{I}(k, s) d s \\
& \leq \text { const. } e^{-|k|(t-1)}|k| \int_{1}^{\frac{t+1}{2}}(1+|k|)|k| \sigma s e^{|k| \sigma} \mu_{1}^{I}(k, s) d s \\
& + \text { const. } e^{-|k|(t-1)}|k| \int_{\frac{t+1}{2}}^{t}\left(1+|k|+\left(|k|^{1 / 2}+|k|\right) \sigma\right) e^{|k| \sigma} \mu_{1}^{I}(k, s) d s \\
& \leq \text { const. }\left(\frac{1}{t^{2}} \bar{\mu}_{\alpha^{\prime}-1}(k, t)+\frac{1}{t^{7 / 2}} \bar{\mu}_{\alpha^{\prime}-1}(k, t)+\frac{1}{t^{4}} \tilde{\mu}_{\alpha^{\prime}-1}(k, t)\right),
\end{aligned}
$$

which shows that $\hat{\psi}_{1}^{r, 2} \in \mathcal{B}_{\alpha^{\prime \prime}, 2,4}$. 
For $\hat{\psi}_{2}^{r, 2}$ we split the integration interval into two sub-intervals, $\left[1, t^{\rho}\right]$ and $\left[t^{\rho}, t\right]$, with $0<\rho<1$. We also rewrite the integral over the first sub-interval using (108), so that

$$
\begin{aligned}
\hat{\psi}_{2}^{r, 2} & =\frac{1}{2} e^{-|k| t} \int_{1}^{t}\left(\left(h_{1,1}(k, \sigma)+2 \kappa \sigma\right) \hat{Q}_{1}(k, s)+(2|k|+i k \sigma) \sigma \hat{Q}_{1}(0, s)\right) d s \\
& =\frac{1}{2} e^{-|k| t} \int_{1}^{t^{\rho}}\left(\left(h_{1,1}(k, \sigma)+2 \kappa \sigma+(2|k|+i k \sigma) \sigma\right) \hat{Q}_{1}(k, s)\right) d s \\
& -\frac{1}{2} e^{-|k| t} \int_{1}^{t^{\rho}}(2|k|+i k \sigma) \sigma k \partial_{k} \hat{Q}_{1}(\zeta, s) d s \\
& +\frac{1}{2} e^{-|k| t} \int_{t^{\rho}}^{t}\left(\left(h_{1,1}(k, \sigma)+2 \kappa \sigma\right) \hat{Q}_{1}(k, s)+(2|k|+i k \sigma) \sigma \hat{Q}_{1}(0, s)\right) d s .
\end{aligned}
$$

For (82) we have, using Proposition 22 with the bound (81) and Proposition 23, with (80),

$$
\begin{aligned}
& \left|\frac{1}{2} e^{-|k| t} \int_{1}^{t^{\rho}}\left(\left(h_{1,1}(k, \sigma)+2 \kappa \sigma+(2|k|+i k \sigma) \sigma\right) \hat{Q}_{1}(k, s)\right) d s\right| \\
& \leq \text { const. } e^{-|k| t} \int_{1}^{t}\left|h_{1,1}(k, \sigma)+2 \kappa \sigma+(2|k|+i k \sigma) \sigma\right| \mu_{1}^{I}(k, s) d s \\
& \leq \text { const. } e^{-|k| t} \int_{1}^{\frac{t+1}{2}}\left(|k|^{3 / 2}+|k|^{3}\right) \sigma^{2} s e^{|k| \sigma} \mu_{1}^{I}(k, s) d s \\
& + \text { const. } e^{-|k| t} \int_{\frac{t+1}{2}}^{t}\left(1+|k|+|k|^{1 / 2} \sigma+|k| \sigma s\right) e^{|k| \sigma} \mu_{1}^{I}(k, s) d s \\
& \leq \text { const. }\left(\frac{1}{t^{3 / 2-\delta}} \bar{\mu}_{\alpha^{\prime}}(k, t)+\frac{1}{t^{2}} \bar{\mu}_{\alpha^{\prime}}(k, t)+\frac{1}{t^{2}} \tilde{\mu}_{\alpha^{\prime}}(k, t)\right) \in \mathcal{B}_{\alpha^{\prime}, \frac{3}{2}-\delta, 2} .
\end{aligned}
$$

For (83) we have, using (115),

$$
\begin{aligned}
& \left|\frac{1}{2} e^{-|k| t} \int_{1}^{t^{\rho}}(2|k|+i k \sigma) \sigma k \partial_{k} \hat{Q}_{1}(\zeta, s) d s\right| \\
& \leq \text { const. } e^{-|k| t}|k|^{2} \int_{1}^{t^{\rho}} s \sigma \frac{1}{s^{2}} d s \\
& \leq \text { const. } e^{-|k| t}|k|^{2}\left(t^{\rho}+\log (1+t)-1\right) \in \mathcal{B}_{\alpha^{\prime}, 2-\rho, \infty} .
\end{aligned}
$$

For (84), we have

$$
\begin{aligned}
& \left|\frac{1}{2} e^{-|k| t} \int_{t^{\rho}}^{t}\left(\left(h_{1,1}(k, \sigma)+2 \kappa \sigma\right) \hat{Q}_{1}(k, s)+(2|k|+i k \sigma) \sigma \hat{Q}_{1}(0, s)\right) d s\right| \\
& \leq \text { const. } e^{-|k| t} \int_{t^{\rho}}^{t}\left|\left(h_{1,1}(k, \sigma)+2 \kappa \sigma\right) \hat{Q}_{1}(k, s)\right| d s+\text { const. } e^{-|k| t}|k| \frac{1}{t^{\rho}},
\end{aligned}
$$

where the second term is in $\mathcal{B}_{\alpha^{\prime}, 1+\rho, \infty}$ by (115). We split the remaining integral into two sub-intervals after setting $\rho \leq 1 / 2$, and make use of (79) and (78), respectively. We get, using Proposition 23 to bound the second integral,

$$
\begin{aligned}
& e^{-|k| t} \int_{t^{\rho}}^{t}\left|\left(h_{1,1}(k, \sigma)+2 \kappa \sigma\right) \hat{Q}_{1}(k, s)\right| d s \\
& =e^{-|k| t} \int_{t^{\rho}}^{\frac{t+1}{2}}\left(|k|^{1 / 2}+|k|^{2}\right) \sigma e^{|k| \sigma} \mu_{1}^{I}(k, s) d s+e^{-|k| t} \int_{\frac{t+1}{2}}^{t}\left(1+|k|+\left(|k|^{1 / 2}+|k|\right) \sigma\right) e^{|k| \sigma} \mu_{1}^{I}(k, s) d s \\
& \leq \text { const. }\left(e^{-|k| t / 2}\left(|k|^{1 / 2}+|k|^{2}\right) \frac{1}{t^{2 \rho}}+\frac{1}{t^{5 / 2}} \bar{\mu}_{\alpha^{\prime}}(k, t)+\frac{1}{t^{5 / 2}} \tilde{\mu}_{\alpha^{\prime}}(k, t)\right),
\end{aligned}
$$

where (115) allows to bound the first term, so that this expression is in $\mathcal{B}_{\alpha^{\prime}, 2 \rho+\frac{1}{2}, \frac{5}{2}}$. Therefore, if we chose $\rho=1 / 2$, then $\hat{\psi}_{2}^{r, 2} \in \mathcal{B}_{\alpha^{\prime}, \frac{3}{2}-\delta, \frac{5}{2}}$. 
To bound $\hat{\psi}_{3}^{r, 2}$ we note that

$$
\begin{aligned}
\left|\hat{\psi}_{3}^{r, 2}\right| & =\left|e^{-|k| t} \int_{1}^{t} \sqrt{-i k} \sigma \hat{Q}_{1}(0, s) d s-e^{-|k|(t-1)} \int_{1}^{t} \kappa \sigma \hat{Q}_{1}(k, s) d s\right| \\
& \leq\left|\left(e^{-|k| t}-e^{-|k|(t-1)}\right) \int_{1}^{t} \kappa \sigma \hat{Q}_{1}(k, s) d s\right| \\
& +\left|e^{-|k| t} \int_{1}^{t}(\sqrt{-i k}-\kappa) \sigma \hat{Q}_{1}(k, s) d s\right| \\
& +\left|e^{-|k| t} \int_{1}^{t} \sqrt{-i k} k \sigma \partial_{k} \hat{Q}_{1}(\zeta, s) d s\right| \\
& \leq \text { const. } e^{-|k|(t-1)}|k| \int_{1}^{t}\left(|k|^{1 / 2}+|k|\right) e^{|k| \sigma} \sigma \mu_{1}^{I}(k, s) d s \\
& + \text { const. } e^{-|k| t}|k|^{3 / 2}+\text { const. } e^{-|k| t}|k|^{3 / 2} \log (1+t)
\end{aligned}
$$

which, using Propositions 22 and 23, (110), and when necessary (114) for the first term and (115) for the other two terms, shows that $\hat{\psi}_{3}^{r, 2} \in \mathcal{B}_{\alpha^{\prime \prime}, \frac{3}{2}-\delta, 5}$.

To bound $\hat{\psi}_{4}^{r, 2}$ we simply integrate with respect to $s$ and then apply (115),

$$
\begin{aligned}
\left|\hat{\psi}_{4}^{r, 2}\right| & =\left|\frac{1}{2} e^{-|k| t} \int_{t}^{\infty}(2 \sqrt{-i k}+2|k|+i k \sigma) \sigma \hat{Q}_{1}(0, s) d s\right| \\
& \leq \text { const. } e^{-|k| t}\left(|k|^{1 / 2}+|k|\right) \frac{1}{t^{2}}+e^{-|k| t}|k| \frac{1}{t^{1}} \in \mathcal{B}_{\alpha^{\prime}, 2, \infty} .
\end{aligned}
$$

Gathering the bounds on the $\hat{\psi}_{i}^{r, 2}$ yields (76), and by the opening remark of the proof also (77).

\subsection{Final improvement of the bounds on $\hat{Q}_{0}, \hat{Q}_{1}$, and $\partial_{k} \hat{Q}_{1}$}

Using Proposition 12, (42), (67) and (76), we get

$$
\begin{aligned}
\hat{\omega} * \hat{\omega} & \in \mathcal{B}_{\alpha^{\prime}, 6,4}, \\
\hat{\psi} *\left(\hat{\omega}-\hat{\omega}_{\mathrm{as}, 1}\right) & \in \mathcal{B}_{\alpha^{\prime \prime}, \frac{9}{2}, \infty} \\
\left(\hat{\psi}-\hat{\psi}_{\mathrm{as}, 1}-\hat{\psi}_{\mathrm{as}, 2}\right) * \hat{\omega}_{\mathrm{as}, 1} & \in \mathcal{B}_{\alpha^{\prime \prime}, \frac{9}{2}-\delta, \infty} .
\end{aligned}
$$

For the term $\left(\hat{\psi}_{\mathrm{as}, 1}+\hat{\psi}_{\mathrm{as}, 2}\right) * \hat{\omega}_{\mathrm{as}, 1}$ we can proceed exactly as in Section 4.5, thanks to the fact that

$$
\sup _{z \in \mathbb{R}}\left\{|z|^{n+2}\left|\psi_{2}^{(n)}(z)\right|\right\}=\text { const. }<\infty, n \geq 0 .
$$

We conclude that

$$
\left(\hat{\psi}_{\mathrm{as}, 1}+\hat{\psi}_{\mathrm{as}, 2}\right) * \hat{\omega}_{\mathrm{as}, 1} \in \mathcal{B}_{\alpha^{\prime}, \infty, 4}
$$

and therefore

$$
\hat{Q}_{1} \in \mathcal{B}_{\alpha^{\prime \prime}, \frac{9}{2}-\delta, 4}
$$

Similarly, we have

$$
\begin{aligned}
\hat{Q}_{0} & \in \mathcal{B}_{\alpha^{\prime \prime}, \frac{9}{2}-\delta, 3}, \\
\partial_{k} \hat{Q}_{1} & \in \mathcal{B}_{\alpha^{\prime \prime}, \frac{5}{2}-\delta, 2} .
\end{aligned}
$$

In the light of (85), we define

$$
\mu_{1}^{I I}:=\frac{1}{s^{9 / 2-\delta}} \bar{\mu}_{\alpha^{\prime \prime}}(k, s)+\frac{1}{s^{4}} \tilde{\mu}_{\alpha^{\prime \prime}}(k, s)
$$

to replace (66) from now on. 


\subsection{Second order in $\hat{\eta}$ and $\hat{\omega}$}

Applying the new bound (85) for $\hat{Q}_{1}$ in a straightforward manner, and in view of Proposition 12 and Remark 9. we find that the second order terms of $\hat{\eta}$ and $\hat{\omega}$ are to be extracted from $\hat{\eta}_{1,1}-\hat{\eta}_{\text {as, } 1}$ and $\hat{\omega}_{1,1}-\hat{\omega}_{\text {as }, 1}$, respectively. Inspecting the limits of these quantities motivates us, in a similar way as in the case of the leading order of $\hat{\eta}$ and $\hat{\omega}$, to define the functions

$$
\begin{gathered}
\hat{\eta}_{\mathrm{as}, 2}(k, t)=-c_{1} \frac{|k|-i k}{\sqrt{-i k}} e^{-\sqrt{-i k} t}, \\
\hat{\omega}_{\mathrm{as}, 2}(k, t)=c_{1}(|k|-i k) e^{-\sqrt{-i k} t},
\end{gathered}
$$

with $c_{1}$ as defined by (29). Note that $\hat{\eta}_{\mathrm{as}, 2} \in \mathcal{B}_{\alpha^{\prime}, \infty, 1}$ and $\hat{\omega}_{\mathrm{as}, 2} \in \mathcal{B}_{\alpha^{\prime}, \infty, 2}$. We now show that

$$
\begin{gathered}
\hat{\eta}_{1,1}-\hat{\eta}_{\mathrm{as}, 1}-\hat{\eta}_{\mathrm{as}, 2} \in \mathcal{B}_{\alpha^{\prime \prime}-1, \frac{5}{2}-\delta, 2-\delta}, \\
\hat{\omega}_{1,1}-\hat{\omega}_{\mathrm{as}, 1}-\hat{\omega}_{\mathrm{as}, 2} \in \mathcal{B}_{\alpha^{\prime \prime}-1, \frac{7}{2}-\delta, 3-\delta} .
\end{gathered}
$$

Remark 14 The bounds (86) and (85) for $\hat{Q}_{0}$ and $\hat{Q}_{1}$, respectively, also show that $\hat{\omega}_{2,1} \in \mathcal{B}_{\alpha^{\prime \prime}, \infty, 3}$, using (113) and $\hat{\omega}_{3,1} \in \mathcal{B}_{\alpha^{\prime \prime}, \frac{7}{2}-\delta, 3}$. This means, as is already mentioned in Remark 9 , that only $\hat{\omega}_{1,1}$ plays a role in (7).

Proof. As for the leading order term, we have

$$
\hat{\omega}_{1,1}-\hat{\omega}_{\mathrm{as}, 1}=\frac{i k}{\kappa}\left(\hat{\eta}_{1,1}-\hat{\eta}_{\mathrm{as}, 1}\right)
$$

so that for the same reasons, the $\mathcal{B}_{\alpha, p, q}$ space of the second order term of $\hat{\omega}$ has indices $p$ and $q$ greater by 1 than that of the second order term of $\hat{\eta}$, and thus we only present the proof for $\hat{\eta}$.

In order to prove (91) we analyze

$$
\begin{aligned}
\hat{\eta}_{1,1}(k, t)-\hat{\eta}_{\mathrm{as}, 1}(k, t)-\hat{\eta}_{\mathrm{as}, 2}(k, t) & =\frac{1}{2} e^{-\kappa(t-1)} \int_{1}^{t} g_{1,1}(k, s-1) \hat{Q}_{1}(k, s) d s \\
& +\frac{1}{2} e^{-\sqrt{-i k} t} \int_{1}^{\infty} 2\left(1+\frac{|k|-i k}{\sqrt{-i k}}\right)(s-1) \hat{Q}_{1}(0, s) d s .
\end{aligned}
$$

We rewrite this expression as a sum of terms which can easily be bounded. Namely,

$$
\hat{\eta}_{1,1}(k, t)-\hat{\eta}_{\mathrm{as}, 1}(k, t)-\hat{\eta}_{\mathrm{as}, 2}(k, t)=\sum_{i=1}^{6} \hat{\eta}_{i}^{r, 2}
$$

with

$$
\begin{aligned}
& \hat{\eta}_{1}^{r, 2}=\frac{1}{2}\left(e^{-\kappa(t-1)}-e^{-\kappa t}\right) \int_{1}^{t}\left(g_{1,1}(k, \sigma)-2 \frac{\kappa}{i k} \kappa \sigma\right) \hat{Q}_{1}(k, s) d s, \\
& \hat{\eta}_{2}^{r, 2}=\frac{1}{2} e^{-\kappa t} \int_{1}^{t}\left(\left(g_{1,1}(k, \sigma)-2 \frac{\kappa}{i k} \kappa \sigma\right) \hat{Q}_{1}(k, s)+\frac{2|k|}{\sqrt{-i k}} \sigma \hat{Q}_{1}(0, s)\right) d s, \\
& \hat{\eta}_{3}^{r, 2}=\left(e^{-\kappa(t-1)}-e^{-\kappa t}\right) \int_{1}^{t} \frac{\kappa}{i k} \kappa \sigma \hat{Q}_{1}(k, s) d s+e^{-\sqrt{-i k} t} \int_{1}^{t} \frac{-i k}{\sqrt{-i k}} \sigma \hat{Q}_{1}(0, s) d s \\
& \hat{\eta}_{4}^{r, 2}=e^{-\kappa t} \int_{1}^{t}\left(\frac{\kappa}{i k} \kappa \hat{Q}_{1}(k, s)+\hat{Q}_{1}(0, s)\right) \sigma d s \\
& \hat{\eta}_{5}^{r, 2}=-\left(e^{-\kappa t}-e^{-\sqrt{-i k} t}\right) \int_{1}^{t}\left(1+\frac{|k|}{\sqrt{-i k}}\right) \sigma \hat{Q}_{1}(0, s) d s \\
& \hat{\eta}_{6}^{r, 2}=e^{-\sqrt{-i k} t} \int_{t}^{\infty}\left(1+\frac{|k|-i k}{\sqrt{-i k}}\right) \sigma \hat{Q}_{1}(0, s) d s .
\end{aligned}
$$

The term $\hat{\eta}_{1}^{r, 2}$ must be bounded by Propositions 19 and 20 for $|k| \leq 1$ and $|k|>1$ separately. We use the bounds

$$
\left|g_{1,1}(k, \sigma)-2 \frac{\kappa^{2}}{i k} \sigma\right| \leq\left\{\begin{array}{ll}
\text { const. } \sigma e^{\left|\Lambda_{-}\right| \sigma} \min \left\{1,\left|\Lambda_{-}\right|(\sigma+1)\right\} & \text { for }|k| \leq 1 \\
\text { const. } e^{\left|\Lambda_{-}\right| \sigma} \min \left\{\left(1+\left|\Lambda_{-}\right| s\right),\left|\Lambda_{-}\right|^{2} \sigma(\sigma+1)\right\} & \text { for }|k|>1
\end{array} .\right.
$$


which can easily be obtained from (95). For $|k| \leq 1$ we have

$$
\begin{aligned}
\left|\hat{\eta}_{1}^{r, 2}\right| & =\left|\frac{1}{2}\left(e^{-\kappa(t-1)}-e^{-\kappa t}\right) \int_{1}^{t}\left(g_{1,1}(k, \sigma)-2 \frac{\kappa}{i k} \kappa \sigma\right) \hat{Q}_{1}(k, s) d s\right| \\
& \leq \text { const. } e^{\Lambda_{-}(t-1)}\left|\Lambda_{-}\right| \int_{1}^{\frac{t+1}{2}}\left|\Lambda_{-}\right| \sigma s e^{\left|\Lambda_{-}\right| \sigma} \mu_{1}^{I I}(k, s) d s \\
& + \text { const. } e^{\Lambda_{-}(t-1)}\left|\Lambda_{-}\right| \int_{\frac{t+1}{2}}^{t} \sigma e^{\left|\Lambda_{-}\right| \sigma} \mu_{1}^{I I}(k, s) d s \\
& \leq \text { const. }\left(\frac{1}{t^{2}} \tilde{\mu}_{\alpha^{\prime \prime}}(k, t)+\frac{1}{t^{7 / 2-\delta}} \bar{\mu}_{\alpha^{\prime \prime}}(k, t)+\frac{1}{t^{3}} \tilde{\mu}_{\alpha^{\prime \prime}}(k, t)\right),
\end{aligned}
$$

and for $|k|>1$, using (114) to deal with the spurious $\left|\Lambda_{-}\right|$factor,

$$
\begin{aligned}
\left|\hat{\eta}_{1}^{r, 2}\right| & =\left|\frac{1}{2}\left(e^{-\kappa(t-1)}-e^{-\kappa t}\right) \int_{1}^{t}\left(g_{1,1}(k, \sigma)-2 \frac{\kappa}{i k} \kappa \sigma\right) \hat{Q}_{1}(k, s) d s\right| \\
& \leq \text { const. } e^{\Lambda_{-}(t-1)}\left|\Lambda_{-}\right| \int_{1}^{\frac{t+1}{2}}\left|\Lambda_{-}\right|^{2} \sigma s e^{\left|\Lambda_{-}\right| \sigma} \mu_{1}^{I I}(k, s) d s \\
& + \text { const. } e^{\Lambda_{-}(t-1)}\left|\Lambda_{-}\right| \int_{\frac{t+1}{2}}^{t}\left(1+\left|\Lambda_{-}\right| s\right) e^{\left|\Lambda_{-}\right| \sigma} \mu_{1}^{I I}(k, s) d s \\
& \leq \text { const. }\left(\frac{1}{t^{4}} \tilde{\mu}_{\alpha^{\prime \prime}-1}(k, t)+\frac{1}{t^{9 / 2-\delta}} \bar{\mu}_{\alpha^{\prime \prime}-1}(k, t)+\frac{1}{t^{4}} \tilde{\mu}_{\alpha^{\prime \prime}-1}(k, t)\right) .
\end{aligned}
$$

This shows that $\hat{\eta}_{1}^{r, 2}$ is in $\mathcal{B}_{\alpha^{\prime \prime}-1, \frac{7}{2}-\delta, 2}$.

For $\hat{\eta}_{2}^{r, 2}$ we use the fact that, using (108),

$$
\begin{aligned}
& \left(g_{1,1}(k, \sigma)-2 \frac{\kappa}{i k} \kappa \sigma\right) \hat{Q}_{1}(k, s)+\frac{2|k|}{\sqrt{-i k}} \sigma \hat{Q}_{1}(0, s) \\
& =\left(g_{1,1}(k, \sigma)-2 \frac{\kappa}{i k} \kappa \sigma+\frac{2|k|}{\sqrt{-i k}} \sigma\right) \hat{Q}_{1}(k, s)-\frac{2|k|}{\sqrt{-i k}} \sigma k \partial_{k} \hat{Q}_{1}(\zeta, s),
\end{aligned}
$$

for some $\zeta \in[0, k]$. We analyze the expression

$$
g_{1,1}(k, \sigma)-2 \frac{\kappa}{i k} \kappa \sigma+\frac{2|k|}{\sqrt{-i k}} \sigma=\frac{\kappa}{i k}\left(e^{\kappa \sigma}+\frac{(|k|+\kappa)^{2}}{i k} e^{-\kappa \sigma}-2 \frac{|k|(|k|+\kappa)}{i k} e^{-|k| \sigma}-2 \kappa \sigma-\frac{2|k| \sqrt{-i k}}{\kappa} \sigma\right)
$$

in some more detail. A straightforward bound is

$$
\begin{aligned}
& \left|g_{1,1}(k, \sigma)-2 \frac{\kappa}{i k} \kappa \sigma+\frac{2|k|}{\sqrt{-i k}} \sigma\right| \\
& =\left|\frac{\kappa}{i k}\left(e^{\kappa \sigma}+\frac{(|k|+\kappa)^{2}}{i k} e^{-\kappa \sigma}-2 \frac{|k|(|k|+\kappa)}{i k} e^{-|k| \sigma}-2 \kappa \sigma-\frac{2|k| \sqrt{-i k}}{\kappa}\right)\right| \\
& \leq\left|\frac{\kappa}{i k}\right|\left|\left(e^{\kappa \sigma}-1\right)-\left(e^{-\kappa \sigma}-1\right)+\frac{2|k|^{2}+2|k| \kappa}{i k}\left(\left(e^{-\kappa \sigma}-1\right)-\left(e^{-|k| \sigma}-1\right)\right)\right| \\
& +\left|\frac{\kappa}{i k}\right|\left|2 \kappa \sigma+\frac{2|k| \sqrt{-i k}}{\kappa} \sigma\right| \leq\left\{\begin{array}{ll}
\text { const. }\left(1+\left|\Lambda_{-}\right|\right) \sigma e^{\left|\Lambda_{-}\right| \sigma} & \text { for }|k| \leq 1 \\
\text { const. }\left(1+\left|\Lambda_{-}\right|(\sigma+1)\right) e^{\left|\Lambda_{-}\right| \sigma} & \text { for }|k|>1
\end{array} .\right.
\end{aligned}
$$

but we may also cancel leading order terms so that

$$
\begin{aligned}
& g_{1,1}(k, \sigma)-2 \frac{\kappa}{i k} \kappa \sigma+\frac{2|k|}{\sqrt{-i k}} \sigma \\
& =\frac{\kappa}{i k}\left(\left(e^{\kappa \sigma}-1-\kappa \sigma-\frac{1}{2} \kappa^{2} \sigma^{2}\right)-\left(e^{-\kappa \sigma}-1+\kappa \sigma-\frac{1}{2} \kappa^{2} \sigma^{2}\right)\right) \\
& +\frac{\kappa}{i k}\left(\frac{2|k|^{2}+2|k| \kappa}{i k}\left(\left(e^{-\kappa \sigma}-1+\kappa \sigma\right)-\left(e^{-|k| \sigma}-1+|k| \sigma\right)\right)\right) \\
& +\frac{\kappa}{i k}\left(\frac{2|k|^{2}+2|k| \kappa}{i k}(|k|-\kappa) \sigma-\frac{2|k| \sqrt{-i k}}{\kappa} \sigma\right),
\end{aligned}
$$


where the third term reduces to $-2 i|k|(\kappa-\sqrt{-i k}) \sigma / k$. This yields

$$
\left|g_{1,1}(k, \sigma)-2 \frac{\kappa}{i k} \kappa \sigma+\frac{2|k|}{\sqrt{-i k}} \sigma\right| \leq\left\{\begin{array}{ll}
\text { const. }\left|\Lambda_{-}\right|^{2} \sigma\left(\sigma^{2}+\sigma+1\right) e^{\left|\Lambda_{-}\right| \sigma} & \text { for }|k| \leq 1 \\
\text { const. }\left|\Lambda_{-}\right|^{3} \sigma\left(\sigma^{2}+\sigma+1\right) e^{\left|\Lambda_{-}\right| \sigma} & \text { for }|k|>1
\end{array} .\right.
$$

Collecting (93) and (94) we have

$$
\begin{aligned}
& \left|g_{1,1}(k, \sigma)-2 \frac{\kappa}{i k} \kappa \sigma+\frac{2|k|}{\sqrt{-i k}} \sigma\right| \\
& \leq\left\{\begin{array}{ll}
\text { const. } \min \left\{\left(1+\left|\Lambda_{-}\right|\right),\left|\Lambda_{-}\right|^{2}\left(\sigma^{2}+\sigma+1\right)\right\} \sigma e^{\left|\Lambda_{-}\right| \sigma} & \text { for }|k| \leq 1 \\
\text { const. } \min \left\{\left(1+\left|\Lambda_{-}\right|(\sigma+1)\right),\left|\Lambda_{-}\right|^{3} \sigma\left(\sigma^{2}+\sigma+1\right)\right\} e^{\left|\Lambda_{-}\right| \sigma} & \text { for }|k|>1
\end{array} .\right.
\end{aligned}
$$

We can now bound $\hat{\eta}_{2}^{r, 2}$ by splitting it into two terms. We have

$$
\begin{aligned}
\left|\hat{\eta}_{2}^{r, 2}\right| & =\left|\frac{1}{2} e^{-\kappa t} \int_{1}^{t}\left(\left(g_{1,1}(k, \sigma)-2 \frac{\kappa}{i k} \kappa \sigma\right) \hat{Q}_{1}(k, s)+\frac{2|k|}{\sqrt{-i k}} \sigma \hat{Q}_{1}(0, s)\right) d s\right| \\
& \leq\left|\frac{1}{2} e^{-\kappa t} \int_{1}^{t}\left(g_{1,1}(k, \sigma)-2 \frac{\kappa}{i k} \kappa \sigma+\frac{2|k|}{\sqrt{-i k}} \sigma\right) \hat{Q}_{1}(k, s) d s\right| \\
& +\left|e^{-\kappa t} \int_{1}^{t} \frac{|k|}{\sqrt{-i k}} \sigma k \partial_{k} \hat{Q}_{1}(\zeta, s) d s\right| .
\end{aligned}
$$

For the term (96) we get, for $|k| \leq 1$,

$$
\begin{aligned}
& \left|\frac{1}{2} e^{-\kappa t} \int_{1}^{t}\left(g_{1,1}(k, \sigma)-2 \frac{\kappa}{i k} \kappa \sigma+\frac{2|k|}{\sqrt{-i k}} \sigma\right) \hat{Q}_{1}(k, s) d s\right| \\
& \leq \text { const. } e^{\Lambda_{-} t} \int_{1}^{\frac{t+1}{2}}\left|\Lambda_{-}\right|^{2}\left(\sigma^{2}+s\right) \sigma e^{\left|\Lambda_{-}\right| \sigma} \mu_{1}^{I I}(k, s) d s \\
& + \text { const. } e^{\Lambda_{-} t} \int_{\frac{t+1}{2}}^{t}\left(1+\left|\Lambda_{-}\right|\right) \sigma e^{\left|\Lambda_{-}\right| \sigma} \mu_{1}^{I I}(k, s) d s \\
& \leq \text { const. }\left(\frac{1}{t^{2-\delta}} \tilde{\mu}_{\alpha^{\prime \prime}}(k, t)+\frac{1}{t^{5 / 2-\delta}} \bar{\mu}_{\alpha^{\prime \prime}}(k, t)+\frac{1}{t^{2}} \tilde{\mu}_{\alpha^{\prime \prime}}(k, t)\right),
\end{aligned}
$$

and for $|k|>1$

$$
\begin{aligned}
& \left|\frac{1}{2} e^{-\kappa t} \int_{1}^{t}\left(g_{1,1}(k, \sigma)-2 \frac{\kappa}{i k} \kappa \sigma+\frac{2|k|}{\sqrt{-i k}} \sigma\right) \hat{Q}_{1}(k, s) d s\right| \\
& \leq \text { const. } e^{\Lambda_{-} t} \int_{1}^{\frac{t+1}{2}}\left|\Lambda_{-}\right|^{3} \sigma\left(\sigma^{2}+s\right) e^{\left|\Lambda_{-}\right| \sigma} \mu_{1}^{I I}(k, s) d s \\
& + \text { const. } e^{\Lambda_{-} t} \int_{\frac{t+1}{2}}^{t}\left(1+\left|\Lambda_{-}\right| s\right) e^{\left|\Lambda_{-}\right| \sigma} \mu_{1}^{I I}(k, s) d s \\
& \leq \text { const. }\left(\frac{1}{t^{3-\delta}} \tilde{\mu}_{\alpha^{\prime \prime}}(k, t)+\frac{1}{t^{7 / 2-\delta}} \bar{\mu}_{\alpha^{\prime \prime}}(k, t)+\frac{1}{t^{3}} \tilde{\mu}_{\alpha^{\prime \prime}}(k, t)\right) .
\end{aligned}
$$

The term (97) is bounded using (116), so that we get

$$
\left|e^{-\kappa t} \int_{1}^{t} \frac{|k| k}{\sqrt{-i k}} \sigma \partial_{k} \hat{Q}_{1}(\zeta, s) d s\right| \leq \text { const. }\left|e^{-\sqrt{-i k} t}\right||k|^{3 / 2} \log (1+t) \in \mathcal{B}_{\alpha^{\prime \prime}, \infty, 3-\delta},
$$

and thus $\hat{\eta}_{2}^{r, 2} \in \mathcal{B}_{\alpha^{\prime \prime}, \frac{5}{2}-\delta, 2-\delta}$.

To bound $\hat{\eta}_{3}^{r, 2}$ we can rearrange the terms and use (108) to get

$$
\begin{aligned}
\hat{\eta}_{3}^{r, 2} & =e^{-\kappa t}\left(e^{\kappa}-1\right) \int_{1}^{t} \frac{\kappa}{i k} \kappa \sigma \hat{Q}_{1}(k, s) d s-e^{-\sqrt{-i k} t} \int_{1}^{t} \frac{i k}{\sqrt{-i k}} \sigma \hat{Q}_{1}(0, s) d s \\
& =\left(e^{-\kappa t}-e^{-\sqrt{-i k} t}\right) \int_{1}^{t} \frac{i k}{\sqrt{-i k}} \sigma \hat{Q}_{1}(0, s) d s \\
& +e^{-\kappa t} \int_{1}^{t}\left(\frac{\kappa^{2}}{i k}\left(e^{\kappa}-1\right)-\frac{i k}{\sqrt{-i k}}\right) \sigma \hat{Q}_{1}(k, s) d s \\
& +e^{-\kappa t} \int_{1}^{t} \frac{i k}{\sqrt{-i k}} k \sigma \partial_{k} \hat{Q}_{1}(\zeta, s) d s,
\end{aligned}
$$


for some $\zeta \in[0, k]$. We then get, using for the first term (111),

$$
\begin{aligned}
\left|\hat{\eta}_{3}^{r, 2}\right| & \leq \text { const. }\left|e^{-\sqrt{-i k} t}\right||k|^{3 / 2} t|k|^{1 / 2}\left|\int_{1}^{t} \sigma \hat{Q}_{1}(0, s) d s\right| \\
& + \text { const. }\left|e^{-\kappa t} \int_{1}^{t} \sqrt{-i k} k \sigma \partial_{k} \hat{Q}_{1}(\zeta, s) d s\right| \\
& + \text { const. }\left|e^{-\kappa t} \int_{1}^{t}\left(\frac{\kappa^{2}}{i k}\left(e^{\kappa}-1\right)+\sqrt{-i k}\right) \sigma \hat{Q}_{1}(k, s) d s\right| .
\end{aligned}
$$

For the third term we use

$$
\left|\frac{\kappa^{2}}{i k}\left(e^{\kappa}-1\right)+\sqrt{-i k}\right|=\left|-i k\left(e^{\kappa}-1\right)-\left(e^{\kappa}-1-\kappa\right)-\kappa+\sqrt{-i k}\right|
$$

which is bounded above, using (110), by

$$
\text { const. }\left(|k|\left|\Lambda_{-}\right| e^{\left|\Lambda_{-}\right|}+\left|\Lambda_{-}\right|^{2} e^{\left|\Lambda_{-}\right|}+\min \left\{\left|\Lambda_{-}\right|^{2},\left|\Lambda_{-}\right|^{3}\right\}\right) \leq \text { const. }\left|\Lambda_{-}\right|^{2} e^{\left|\Lambda_{-}\right|} .
$$

We therefore have

$$
\begin{aligned}
\left|\hat{\eta}_{3}^{r, 2}\right| & \leq \text { const. }\left|e^{-\sqrt{-i k} t}\right|\left(|k|^{2} t+|k|^{3 / 2} \log (1+t)\right) \\
& + \text { const. } e^{\Lambda_{-} t} e^{\left|\Lambda_{-}\right|} \int_{1}^{t}\left|\Lambda_{-}\right|^{2} \sigma e^{\left|\Lambda_{-}\right| \sigma} \mu_{1}^{I I}(k, s) d s,
\end{aligned}
$$

which, due to (116), Propositions 19 and 20, and using (114) to trade, where appropriate, one factor of $\left|\Lambda_{-}\right|$for a factor $t^{-1}$, shows that $\hat{\eta}_{3}^{r, 2} \in \mathcal{B}_{\alpha^{\prime \prime}-1,4-\delta, 2}$.

To bound $\hat{\eta}_{4}^{r, 2}$ we rearrange the terms using (108), for some $\zeta \in[0, k]$, such that

$$
\begin{aligned}
\left|\hat{\eta}_{4}^{r, 2}\right| & =\left|e^{-\kappa t} \int_{1}^{t}\left(\frac{\kappa}{i k} \kappa \hat{Q}_{1}(k, s)+\hat{Q}_{1}(0, s)\right) \sigma d s\right| \\
& \leq \text { const. }\left|e^{-\sqrt{-i k} t}\right|\left|\int_{1}^{t}\left(\frac{\kappa^{2}}{i k}+1\right) \sigma \hat{Q}_{1}(0, s) d s\right| \\
& + \text { const. }\left|e^{-\sqrt{-i k} t}\right|\left|\int_{1}^{t} \frac{\kappa^{2}}{i k} k \sigma \partial_{k} \hat{Q}_{1}(\zeta, s) d s\right| \\
& \leq \text { const. }\left|e^{-\sqrt{-i k} t}\right|\left(|k|+\left(|k|+|k|^{2}\right) \log (1+t)\right) .
\end{aligned}
$$

We then use (116) to show that $\hat{\eta}_{4}^{r, 2} \in \mathcal{B}_{\alpha^{\prime \prime}, \infty, 2-\delta}$.

For $\hat{\eta}_{5}^{r, 2}$ we use (111) and (116), so that

$$
\left|\hat{\eta}_{5}^{r, 2}\right|=\left|\left(e^{-\kappa t}-e^{-\sqrt{-i k} t}\right) \int_{1}^{t}\left(1+\frac{|k|}{\sqrt{-i k}}\right) \sigma \hat{Q}_{1}(0, s) d s\right| \leq \text { const. }\left|e^{-\sqrt{-i k} t}\right||k|^{3 / 2} t\left(1+|k|^{1 / 2}\right) \in \mathcal{B}_{\alpha^{\prime \prime}, \infty, 2} .
$$

Finally, using (116) to bound $\hat{\eta}_{6}^{r, 2}$, we get

$$
\left|\hat{\eta}_{6}^{r, 2}\right|=\left|e^{-\sqrt{-i k} t} \int_{t}^{\infty}\left(1+\frac{|k|-i k}{\sqrt{-i k}}\right) \sigma \hat{Q}_{1}(0, s) d s\right| \leq \text { const. }\left|e^{-\sqrt{-i k} t}\right|\left(1+|k|^{1 / 2}\right) \frac{1}{t^{2}} \in \mathcal{B}_{\alpha^{\prime \prime}, \infty, 2}
$$

Gathering the bounds on the $\hat{\eta}_{i}^{r, 2}$ terms yields (91), and by the opening remark of the proof also (92). 


\section{A Appendix}

\section{A.1 Explicit expressions for the asymptotes}

The following are explicit functions for which Theorem 1 is true:

$$
\begin{aligned}
& \varphi_{1}(z)=-\frac{1}{4 \sqrt{\pi}} \frac{r+1-z^{2}+z r+2 z}{r^{3} \sqrt{r+1}}, \\
& \psi_{1}(z)=-\frac{1}{4 \sqrt{\pi}} \frac{r+1-z^{2}-z r-2 z}{r^{3} \sqrt{r+1}}, \\
& \varphi_{2,1}(z)=-\frac{1}{\pi} \frac{2 z}{r^{4}} \\
& \varphi_{2,2}(z)=\frac{1}{2 \pi} \frac{1-z^{2}}{r^{4}}, \\
& \psi_{2,1}(z)=-\frac{1}{\pi} \frac{1-z^{2}}{r^{4}}, \\
& \psi_{2,2}(z)=-\frac{1}{2 \pi} \frac{2 z}{r^{4}} \\
& \eta_{W}(z)=-\frac{1}{2 \sqrt{\pi z^{3}}}\left\{\begin{array}{cc}
e^{-1 / 4 z}, & z \geq 0 \\
0, & z<0
\end{array},\right. \\
& \omega_{W}(z)=\frac{1}{4 \sqrt{\pi z^{5}}}\left\{\begin{array}{ll}
(1-2 z) e^{-1 / 4 z}, & z \geq 0 \\
0, & z<0
\end{array},\right. \\
& \eta_{B}(z)=-\frac{1}{4 \pi z^{3}}\left\{\begin{array}{ll}
2 z+\sqrt{\pi|z|}(1-2 z) e^{-1 / 4 z}(1-\operatorname{erfi}(1 / \sqrt{4|z|})), & z \geq 0 \\
2 z+\sqrt{\pi|z|}(1-2 z) e^{-1 / 4 z}(1-\operatorname{erf}(1 / \sqrt{4|z|})), & z<0
\end{array},\right. \\
& \omega_{B}(z)=\frac{1}{8 \pi z^{4}}\left\{\begin{array}{ll}
2 z(1-4 z)+\sqrt{\pi|z|}(1-6 z) e^{-1 / 4 z}(1-\operatorname{erfi}(1 / \sqrt{4|z|})), & z \geq 0 \\
2 z(1-4 z)+\sqrt{\pi|z|}(1-6 z) e^{-1 / 4 z}(1-\operatorname{erf}(1 / \sqrt{4|z|})), & z<0
\end{array},\right.
\end{aligned}
$$

where

$$
r=\sqrt{1+z^{2}} .
$$

These functions are obtained by taking the inverse Fourier transform of the asymptotic terms calculated in Section 4 ,

\section{A.2 Technical aspects of computations}

\section{Mean-value theorem applied to $\hat{Q}_{1}$}

Applying the mean-value theorem in the variable $k$ we have

$$
\hat{Q}_{1}(k, s)=\hat{Q}_{1}(0, s)+k \partial_{k} \hat{Q}_{1}(\zeta, s),
$$

with some $\zeta \in[0, k]$ and (see [1])

$$
\partial_{k} \hat{Q}_{1} \in \mathcal{B}_{\alpha, \frac{3}{2}, 2} .
$$

The bound on $\partial_{k} \hat{Q}_{1}$ is improved in Sections 4.5.1 and 4.7, where it is proved that this function is in $\mathcal{B}_{\alpha^{\prime \prime}, 2,2}$ and $\mathcal{B}_{\alpha^{\prime \prime}, \frac{5}{2}-\delta, 2}$, respectively.

\section{Inequalities for $k$ and $\kappa$}

Since $\kappa=\sqrt{k^{2}-i k}$ and $\Lambda_{-}=-\operatorname{Re}(\kappa)=-\frac{1}{2} \sqrt{2 \sqrt{k^{2}+k^{4}}+2 k^{2}}$, we have

$$
|\kappa|=\left(k^{2}+k^{4}\right)^{1 / 4} \leq|k|^{1 / 2}+|k| \leq 2^{3 / 4}|\kappa| \leq 2^{3 / 4}(1+|k|),
$$

and that

$$
|k| \leq\left|\Lambda_{-}\right| \leq|\kappa| \leq \sqrt{2}\left|\Lambda_{-}\right|
$$

from which we get, for $\sigma \geq 0$,

$$
e^{\Lambda_{-} \sigma} \leq e^{-|k| \sigma} .
$$


The following inequalities are used throughout the proofs

$$
\begin{aligned}
|\kappa-\sqrt{-i k}| & =\left|\frac{k^{2}-i k-(-i k)}{\sqrt{k^{2}-i k}+\sqrt{-i k}}\right| \leq \frac{k^{2}}{2|\sqrt{-i k}|} \\
& \leq \text { const. }|k|^{3 / 2} \leq \text { const. } \min \left\{\left|\Lambda_{-}\right|^{2},\left|\Lambda_{-}\right|^{3}\right\},
\end{aligned}
$$

and

$$
\begin{aligned}
\left|e^{-\kappa t}-e^{-\sqrt{-i k} t}\right| & \leq\left|e^{-\sqrt{-i k} t}\left(e^{(\sqrt{-i k}-\kappa) t}-1\right)\right| \\
& \leq \text { const. }\left|e^{-\sqrt{-i k} t}\right||\sqrt{-i k}-\kappa| t \\
& \leq \text { const. }\left|e^{-\sqrt{-i k} t}\right||k|^{3 / 2} t
\end{aligned}
$$

Some inequalities for $\bar{\mu}_{\alpha}$ and $\tilde{\mu}_{\alpha}$

Using the notation introduced in Definition 4, we have for $\alpha \geq 0$ and $1 \leq t<2$,

$$
\begin{aligned}
& \bar{\mu}_{\alpha}(k, t) \leq \text { const. } \tilde{\mu}_{\alpha}(k, t) \leq \text { const. } \\
& \tilde{\mu}_{\alpha}(k, t) \leq \text { const. } \bar{\mu}_{\alpha}(k, t) \leq \text { const. }
\end{aligned}
$$

and that for $t \geq 2$ and $\beta \geq 0$,

$$
\begin{aligned}
e^{-|k|(t-1)} \mu_{\alpha, r}(k, t) & \leq \text { const. } e^{-|k|(t-1)} \leq \text { const. } \bar{\mu}_{\beta}(k, t), \\
e^{\Lambda_{-}(t-1)} \mu_{\alpha, r}(k, t) & \leq \text { const. } e^{\Lambda_{-}(t-1)} \leq \text { const. } \tilde{\mu}_{\beta}(k, t),
\end{aligned}
$$

such that we have, for all $t \geq 0$,

$$
\begin{aligned}
e^{-|k|(t-1)} \mu_{\alpha, r}(k, t) & \leq \text { const. } \bar{\mu}_{\alpha}(k, t), \\
e^{\Lambda_{-}(t-1)} \mu_{\alpha, r}(k, t) & \leq \text { const. } \tilde{\mu}_{\alpha}(k, t) .
\end{aligned}
$$

Another important inequality used in the proofs is that, for $p \geq 0$,

$$
|k|^{p} \mu_{\alpha, r}(k, t) \leq \frac{\text { const. }}{t^{r p}} \mu_{\alpha-p, r}(k, t),
$$

which is due to the fact that

$$
|k|^{p} \mu_{\alpha, r}(k, t)=\frac{t^{r p}}{t^{r p}} \frac{|k|^{p}}{1+\left(|k| t^{r}\right)^{\alpha}} \leq \frac{\text { const. }}{t^{r p}} \frac{1}{1+\left(|k| t^{r}\right)^{\alpha-p}} .
$$

\section{Function spaces for some exponential functions}

Proposition 15 For $\alpha \geq 1, p, q \geq 0$, we have

$$
\begin{aligned}
k^{p} e^{-|k| t} & \in \mathcal{B}_{\alpha, p, \infty}, p \geq 0, \\
k^{q} e^{-\sqrt{-i k t}}, k^{q} e^{-\kappa t} & \in \mathcal{B}_{\alpha, \infty, 2 q}, q \geq 0 .
\end{aligned}
$$

Proof. Using Definition 5 for functions belonging in $\mathcal{B}_{\alpha, p, \infty}$ spaces, we must have

$$
\sup _{t \geq 1} \sup _{k \in \mathbb{R} \backslash\{0\}} \frac{\mid k^{p} e^{-|k| t \mid}}{\frac{1}{t^{p}} \bar{\mu}_{\alpha}(k, t)}=\sup _{t \geq 1} \sup _{k \in \mathbb{R} \backslash\{0\}}(|k| t)^{p}\left(1+(|k| t)^{\alpha}\right) e^{-|k| t}<\infty .
$$

We use the change of variable $z=k t$, so that

$$
\sup _{t \geq 1} \sup _{z \in \mathbb{R} \backslash\{0\}}|z|^{p}\left(1+|z|^{\alpha}\right) e^{-|z|}<\infty .
$$

Similarly we have

$$
\sup _{t \geq 1} \sup _{k \in \mathbb{R} \backslash\{0\}} \frac{\left|k^{q} e^{-|k| t}\right|}{\frac{1}{t^{2 q}} \tilde{\mu}_{\alpha}(k, t)}=\sup _{t \geq 1} \sup _{k \in \mathbb{R} \backslash\{0\}}\left(|k| t^{2}\right)^{q}\left(1+\left(|k| t^{2}\right)^{\alpha}\right)\left|e^{-\sqrt{-i k t^{2}}}\right|,
$$


and using the change of variable $z=k t^{2}$, we get

$$
\sup _{t \geq 1} \sup _{z \in \mathbb{R} \backslash\{0\}}|z|^{q}\left(1+|z|^{\alpha}\right) e^{-\sqrt{|z| / 2}}<\infty .
$$

For the functions $k^{q} e^{-\kappa t}$ we have

$$
\begin{aligned}
\sup _{t \geq 1} \sup _{k \in \mathbb{R} \backslash\{0\}} \frac{\left|k^{q} e^{-\kappa t}\right|}{\frac{1}{t^{2 q}} \tilde{\mu}_{\alpha}(k, t)} & =\sup _{t \geq 1} \sup _{k \in \mathbb{R} \backslash\{0\}}\left(|k| t^{2}\right)^{q}\left(1+\left(|k| t^{2}\right)^{\alpha}\right) e^{\Lambda_{-} t} \\
& \leq \sup _{t \geq 1} \sup _{k \in \mathbb{R} \backslash\{0\}}\left(\left|\Lambda_{-}\right| t\right)^{2 q}\left(1+\left(\left(\left|\Lambda_{-}\right| t\right)^{2 \alpha}\right) e^{\Lambda_{-} t}\right.
\end{aligned}
$$

and with the change of variable $z=\left|\Lambda_{-}\right| t$

$$
\sup _{t \geq 1} \sup _{z \in \mathbb{R} \backslash\{0\}}|z|^{2 q}\left(1+|z|^{2 \alpha}\right) e^{-z}<\infty .
$$

\section{A.3 Bounds on convolution}

We present variants of Proposition 9 and Corollary 10 from [9], which give bounds on convolution products in $\mathcal{B}_{\alpha, p, q}$ spaces.

Proposition 16 (convolution) Let $\alpha>1, s \geq r \geq 0$, and let $a, b$ be continuous functions from $\mathbb{R} \backslash\{0\} \times[1, \infty)$ to $\mathbb{C}$ satisfying the bounds,

$$
\begin{aligned}
|a(k, t)| & \leq \mu_{\alpha, r}(k, t), \\
|b(k, t)| & \leq \mu_{\alpha, s}(k, t),
\end{aligned}
$$

with $\mu_{a, r}$ and $\mu_{\alpha, s}$ as given in Definition 4 . Then, the convolution $a * b$ is a continuous function from $\mathbb{R} \times[1, \infty)$ to $\mathbb{C}$ and we have the bound

$$
|(a * b)(k, t)| \leq \text { const. } \frac{1}{t^{s}} \mu_{\alpha, r}(k, t),
$$

uniformly in $t \geq 1, k \in \mathbb{R}$.

Proof. We begin by splitting the integration interval into three sub-intervals, so that

$$
\begin{aligned}
2 \pi|(a * b)(k, t)| & \leq \int_{-\infty}^{\infty} \mu_{\alpha, r}\left(k^{\prime}, t\right) \mu_{\alpha, s}\left(k-k^{\prime}, t\right) d k^{\prime}= \\
& =\int_{-\infty}^{-k / 2} \ldots d k^{\prime}+\int_{k / 2}^{\infty} \ldots d k^{\prime}+\int_{-k / 2}^{k / 2} \ldots d k^{\prime}
\end{aligned}
$$

where we only consider $k>0$ since the functions $\mu_{\alpha, r}$ and $\mu_{\alpha, s}$ are even with respect to $k$. We first note that

$$
\begin{aligned}
& \int_{-\infty}^{-k / 2} \mu_{\alpha, r}\left(k^{\prime}, t\right) \mu_{\alpha, s}\left(k-k^{\prime}, t\right) d k^{\prime}+\int_{k / 2}^{\infty} \mu_{\alpha, r}\left(k^{\prime}, t\right) \mu_{\alpha, s}\left(k-k^{\prime}, t\right) d k^{\prime} \\
& \leq \text { const. } \mu_{\alpha, r}( \pm k / 2, t) \int_{\mathbb{R}} \mu_{\alpha, s}\left(k-k^{\prime}, t\right) d k^{\prime} \leq \frac{\text { const. }}{t^{s}} \mu_{\alpha, r}(k, t),
\end{aligned}
$$

where the factor $t^{-s}$ arises from the change of variables used in the integral. For $k t^{r} \leq 1$, we have $\frac{1}{2} \leq \mu_{\alpha, r} \leq 1$, so that

$$
\int_{-k / 2}^{k / 2} \mu_{\alpha, r}\left(k^{\prime}, t\right) \mu_{\alpha, s}\left(k-k^{\prime}, t\right) d k^{\prime} \leq \int_{\mathbb{R}} \mu_{\alpha, s}\left(k-k^{\prime}, t\right) d k^{\prime} \leq\left(\text { const. } \mu_{\alpha, r}(k, t)\right) \cdot \frac{\text { const. }}{t^{s}} .
$$

For $k t^{r}>1$, we also have $k t^{s}>1$, and furthermore

$$
\frac{\mu_{\alpha, s}(k, t)}{\mu_{\alpha, r}(k, t)}=\frac{1+\left(|k| t^{r}\right)^{\alpha}}{1+\left(|k| t^{s}\right)^{\alpha}} \leq \frac{2\left(|k| t^{r}\right)^{\alpha}}{\left(|k| t^{s}\right)^{\alpha}}=2 t^{\alpha(r-s)}
$$


which shows that

$$
\int_{-k / 2}^{k / 2} \mu_{\alpha, r}\left(k^{\prime}, t\right) \mu_{\alpha, s}\left(k-k^{\prime}, t\right) d k^{\prime} \leq \mu_{\alpha, s}(k / 2, t) \int_{\mathbb{R}} \mu_{\alpha, r}\left(k^{\prime}, t\right) d k^{\prime} \leq \mu_{\alpha, r}(k / 2, t) 2 t^{\alpha(r-s)} \frac{\text { const. }}{t^{r}},
$$

which, since $\alpha>1$ and $s \geq r$, is bounded by a multiple of $\mu_{\alpha, r}(k, t) / t^{s}$. Gathering the bounds yields (117).

Corollary 17 Let $\alpha_{i}>1$, and, for $i=1,2$ let $p_{i}, q_{i} \geq 0$. Let $\hat{f}_{i} \in \mathcal{B}_{\alpha_{i}, p_{i}, q_{i}}$, and let

$$
\begin{aligned}
\alpha & =\min \left\{\alpha_{1}, \alpha_{2}\right\}, \\
p & =\min \left\{p_{1}+p_{2}+1, p_{1}+q_{2}+2, p_{2}+q_{1}+2\right\}, \\
q & =q_{1}+q_{2}+2 .
\end{aligned}
$$

Then $\hat{f}_{1} * \hat{f}_{2} \in \mathcal{B}_{\alpha, p, q}$ and there exists a constant $C$, dependent only on $\alpha_{i}$, such that

$$
\left\|\hat{f}_{1} * \hat{f}_{2} ; \mathcal{B}_{\alpha, p, q}\right\| \leq C\left\|\hat{f}_{1} ; \mathcal{B}_{\alpha_{1}, p_{1}, q_{1}}\right\| \cdot\left\|\hat{f}_{2} ; \mathcal{B}_{\alpha_{2}, p_{2}, q_{2}}\right\|
$$

Proof. Using that $\mathcal{B}_{\alpha_{i}, p_{i}, q_{i}} \subset \mathcal{B}_{\min \left\{\alpha_{1}, \alpha_{2}\right\}, p_{i}, q_{i}}$, this is an immediate consequence of Proposition 16 .

Proposition 18 (convolution with $|\kappa|^{-1}$ discontinuity) Let $\alpha_{i}>1$, and, for $i=1,2$ let $p_{i}, q_{i} \geq 0$. Let $\hat{f} \in \mathcal{B}_{\alpha_{1}, p_{1}, q_{1}}$ and $\kappa \cdot \hat{g} \in \mathcal{B}_{\alpha_{2}, p_{2}, q_{2}}$, and let

$$
\begin{aligned}
& \alpha=\min \left\{\alpha_{1}, \alpha_{2}\right\}, \\
& p=\min \left\{p_{1}+p_{2}+\frac{1}{2}, p_{1}+q_{2}+1\right\}, \\
& q=\min \left\{q_{1}+p_{2}+\frac{1}{2}, q_{1}+q_{2}+1\right\} .
\end{aligned}
$$

Then $\hat{f} * \hat{g} \in \mathcal{B}_{\alpha, p, q}$ and there exists a constant $C$, dependent only on $\alpha_{i}$, such that

$$
\left\|\hat{f} * \hat{g} ; \mathcal{B}_{\alpha, p, q}\right\| \leq C\left\|\hat{f} ; \mathcal{B}_{\alpha_{1}, p_{1}, q_{1}}\right\| \cdot\left\|\hat{g} ; \mathcal{B}_{\alpha_{2}, p_{2}, q_{2}}\right\|
$$

Proof. This proposition is a consequence of Proposition 11 of [1].

\section{A.4 Convolution with the semi-groups $e^{\Lambda_{-} t}$ and $e^{-|k| t}$}

In an effort of self-consistency, we present the results for the convolution with the semi-groups $e^{\Lambda_{-} t}$ and $e^{-|k| t}$ which are all proved in [9]. In order to bound the integrals over the interval $[1, t]$ we systematically split them into integrals over $\left[1, \frac{1+t}{2}\right]$ and integrals over $\left[\frac{1+t}{2}, t\right]$ and bound the resulting terms separately. The range for the parameter $\beta$ has been extended to include values between 0 and 1 using Hölder's inequality in the propositions for the intervals $[(t+1) / 2, t]$ and $[t, \infty)$. In practice, when a logarithmic bound is found we use that for all $\delta \in(0,1)$ there exists a constant such that

$$
\log (1+t) \leq \text { const. } t^{\delta}
$$

in order to present a bound in terms of $\mathcal{B}_{\alpha, p, q}$ spaces.

For the semi-group $e^{\Lambda_{-} t}$ we have:

Proposition 19 Let $\alpha \geq 0, r \geq 0$ and $\delta \geq 0$ and $\gamma+1 \geq \beta \geq 0$. Then,

$$
\begin{aligned}
& e^{\Lambda_{-}(t-1)} \int_{1}^{\frac{t+1}{2}} e^{\left|\Lambda_{-}\right|(s-1)}\left|\Lambda_{-}\right| \frac{(s-1)^{\gamma}}{s^{\delta}} \mu_{\alpha, r}(k, s) d s \\
& \leq\left\{\begin{array}{l}
\operatorname{const} \cdot \frac{1}{t^{\beta}} \tilde{\mu}_{\alpha}(k, t), \text { if } \delta>\gamma+1 \\
\operatorname{const} \cdot \frac{\log (1+t)}{t^{\beta}} \tilde{\mu}_{\alpha}(k, t), \text { if } \delta=\gamma+1 \\
\operatorname{const} \cdot \frac{t^{\gamma+1-\delta}}{t^{\beta}} \tilde{\mu}_{\alpha}(k, t), \text { if } \delta<\gamma+1
\end{array}\right.
\end{aligned}
$$

uniformly in $t \geq 1$ and $k \in \mathbb{R}$. 
Proposition 20 Let $\alpha \geq 0, r \geq 0, \delta \in \mathbb{R}$, and $\beta \in[0,1]$. Then,

$$
e^{\Lambda_{-}(t-1)} \int_{\frac{t+1}{2}}^{t} e^{\left|\Lambda_{-}\right|(s-1)}\left|\Lambda_{-}\right|^{\beta} \frac{1}{s^{\delta}} \mu_{\alpha, r}(k, s) d s \leq \frac{\text { const. }}{t^{\delta-1+\beta}} \mu_{\alpha, r}(k, t),
$$

uniformly in $t \geq 1$ and $k \in \mathbb{R}$.

Proposition 21 Let $\alpha \geq 0, r \geq 0, \delta>1$, and $\beta \in[0,1]$. Then,

$$
\begin{aligned}
e^{\left|\Lambda_{-}\right|(t-1)} \int_{t}^{\infty} e^{\Lambda_{-}(s-1)}\left|\Lambda_{-}\right|^{\beta} \frac{1}{s^{\delta}} \mu_{\alpha, r}(k, s) d s & \leq \frac{\text { const. }}{t^{\delta-1+\beta}} \mu_{\alpha, r}(k, t), \\
\left|\frac{\kappa}{i k}\left(e^{\left|\Lambda_{-}\right|(t-1)}-e^{\Lambda_{-}(t-1)}\right)\right| \int_{t}^{\infty} e^{\Lambda_{-}(s-1)}\left|\Lambda_{-}\right|^{\beta} \frac{1}{s^{\delta}} \mu_{\alpha, r}(k, s) d s & \leq \frac{\text { const. }}{t^{\delta-2+\beta}} \mu_{\alpha, r}(k, t),
\end{aligned}
$$

uniformly in $t \geq 1$ and $k \in \mathbb{R}$.

The results for the semi-group $e^{-|k| t}$ are very similar.

Proposition 22 Let $\alpha \geq 0, r \geq 0$ and $\delta \geq 0$ and $\gamma+1 \geq \beta \geq 0$. Then,

$$
\begin{aligned}
& e^{-|k|(t-1)} \int_{1}^{\frac{t+1}{2}} e^{|k|(s-1)}|k|^{\beta} \frac{(s-1)^{\gamma}}{s^{\delta}} \mu_{\alpha, r}(k, s) d s \\
& \leq\left\{\begin{array}{l}
\text { const. } \frac{1}{t^{\beta}} \bar{\mu}_{\alpha}(k, t), \text { if } \delta>\gamma+1 \\
\text { const. } \frac{\log (1+t)}{t^{\beta}} \bar{\mu}_{\alpha}(k, t), \text { if } \delta=\gamma+1 \\
\text { const. } \frac{t^{\gamma+1-\delta}}{t^{\beta}} \bar{\mu}_{\alpha}(k, t), \text { if } \delta<\gamma+1
\end{array}\right.
\end{aligned}
$$

uniformly in $t \geq 1$ and $k \in \mathbb{R}$.

Proposition 23 Let $\alpha \geq 0, r \geq 0, \delta \in \mathbb{R}$, and $\beta \in[0,1]$. Then,

$$
e^{-|k|(t-1)} \int_{\frac{t+1}{2}}^{t} e^{|k|(s-1)}|k|^{\beta} \frac{1}{s^{\delta}} \mu_{\alpha, r}(k, s) d s \leq \frac{\text { const. }}{t^{\delta-1+\beta}} \mu_{\alpha, r}(k, t),
$$

uniformly in $t \geq 1$ and $k \in \mathbb{R}$.

Proposition 24 Let $\alpha \geq 0, r \geq 0, \delta>1, \beta \in[0,1]$. Then,

$$
\begin{aligned}
e^{|k|(t-1)} \int_{t}^{\infty} e^{-|k|(s-1)}|k|^{\beta} \frac{1}{s^{\delta}} \mu_{\alpha, r}(k, s) d s & \leq \frac{\text { const. }}{t^{\delta-1+\beta}} \mu_{\alpha, r}(k, t), \\
\left|\frac{|k|}{i k}\left(e^{|k|(t-1)}-e^{-|k|(t-1)}\right)\right| \int_{t}^{\infty} e^{-|k|(s-1)}|k|^{\beta} \frac{1}{s^{\delta}} \mu_{\alpha, r}(k, s) d s & \leq \frac{\text { const. }}{t^{\delta-1+\beta}} \mu_{\alpha, r}(k, t),
\end{aligned}
$$

uniformly in $t \geq 1$ and $k \in \mathbb{R}$.

\section{References}

[1] Christoph Boeckle and Peter Wittwer, Decay estimates for solutions of the two-dimensional NavierStokes equations in the presence of a wall, http://arxiv.org/abs/1104.0619, 2011.

[2] _ Artificial boundary conditions for stationary Navier-Stokes flows past bodies in the halfplane, to appear on arXiv.org, 2012.

[3] Sebastian Bönisch, Vincent Heuveline, and Peter Wittwer, Adaptive boundary conditions for exterior flow problems, Journal of Mathematical Fluid Mechanics 7 (2005), no. 1, 85-107. 
[4] - Second order adaptive boundary conditions for exterior flow problems: Non-symmetric stationary flows in two dimensions, Journal of Mathematical Fluid Mechanics 10 (2008), no. 1, 45-70.

[5] Zhengguang Guo, Peter Wittwer, and Yong Zhou, Leading order asymptotics of stationary NavierStokes flows in the presence of a wall, Mathematical Models and Methods in Applied Sciences 22 (2012), no. 3, 1150018.

[6] Vincent Heuveline and Peter Wittwer, Adaptive boundary conditions for exterior stationary flows in three dimensions, Journal of Mathematical Fluid Mechanics 12 (2010), no. 4, 554-575.

[7] _ Fundamental trends in fluid-structure interaction, Contemporary Challenges in Mathematical Fluid Dynamics and Its Applications, vol. 1, ch. 2. Exterior Flows at Low Reynolds Numbers: Concepts, Solutions, and Applications, pp. 77-169, World Scientific, 2010.

[8] Matthieu Hillairet and Peter Wittwer, On the vorticity of the Oseen problem in a half plane, Physica D - Nonlinear Phenomena 237 (2008), 1388-1421.

[9] _ Existence of stationary solutions of the Navier-Stokes equations in two dimensions in the presence of a wall, Journal of Evolution Equations 9 (2009), no. 4, 675-706.

[10] _ Asymptotic description of solutions of the exterior Navier-Stokes problem in a half space, http://arxiv.org/abs/1107.1028 (accepted for publication in ARMA), 2011.

[11] Jonas Latt, Yannick Grillet, Bastien Chopard, and Peter Wittwer, Simulating an exterior domain for drag force computations in the lattice boltzmann method, Math. Comp. Sim. 72 (2006), 169-172. 